Research Article

\title{
Global Analysis of a Blood Flow Model with Artificial Boundaries
}

\section{S. C. Oukouomi Noutchie}

School of Mathematical Sciences, North-West University, Mafikeng 2735, South Africa

Correspondence should be addressed to S. C. Oukouomi Noutchie; 23238917@nwu.ac.za

Received 3 June 2013; Accepted 4 August 2013

Academic Editors: S. He and K. Karamanos

Copyright ( 2013 S. C. Oukouomi Noutchie. This is an open access article distributed under the Creative Commons Attribution License, which permits unrestricted use, distribution, and reproduction in any medium, provided the original work is properly cited.

\begin{abstract}
A theoretical model for blood flow in ramifying arteries was introduced and studied numerically (Quarteroni and Veneziani, 2003). A special experimental condition was considered on the artificial boundaries. In this paper, the aim is to analyze the well-posedness of this model, with the focus on the stilted boundary conditions. We use Brouwer's fixed point theorem to show the existence of a solution to the stationary problem. For the evolutionary version, we use some energy estimates and Galerkin's method to prove global existence, uniqueness, and stability of a weak solution.
\end{abstract}

\section{Introduction}

Evolution systems with artificial boundaries are difficult to analyze as a result of the complex dynamics at the boundaries and very few papers have attempted to capture these processes from an analytical point of view. This paper explores this question regarding the flow of blood in a portion of a large artery and addresses the analysis of the Navier-Stokes problem, having provided boundary conditions which can be considered as a generalization of the mean pressure drop problem investigated in [1-3], as they arise in bioengineering applications. Our purpose is to consider both the stationary case and the nonstationary case. In this regard, we will prove the existence of a weak solution for the stationary case based on Brouwer's fixed point theorem and afterwards, we will establish a well-posedness analysis for the nonstationary case based on a suitable energy estimate that we are going to derive as well as a well-known compactness argument.

1.1. Basic Notations. In this subsection, we summarize some notations that will occur throughout the paper. Vectors and tensors are denoted by bold-face letters

$\mathbf{x}=\left(x_{1}, x_{2}, x_{3}\right)$ : location of fluid particle,

u: velocity field of the flow,

p: pressure, $\nabla p$ : the pressure gradient,

I: identity tensor,

T: symmetric Cauchy stress tensor,

$\mu$ : dynamic viscosity,

$\rho$ : fluid mass density,

$\mathbb{R}$ : the set of real numbers,

$|\cdot|$ : the absolute value of $\mathbb{R}$ and correspondingly, the norm of $\mathbb{R}^{3}$,

$\Omega$ : a bounded domain in $\mathbb{R}^{3}$,

$\Gamma$ : the boundary of $\Omega$,

$\nabla \mathbf{u}$ : the gradient of $\mathbf{u}$,

$\nabla \cdot \mathbf{u}$ : the divergence of $\mathbf{u}$

$\Delta \mathbf{u}$ : the Laplacian of $\mathbf{u}$.

The spaces $C(\Omega), C^{k}(\Omega), C_{0}^{k}(\Omega)$, and $C_{0}^{\infty}(\Omega)$ and their vector-valued analogues $\mathbf{C}(\Omega), \mathbf{C}^{k}(\Omega), \mathbf{C}_{0}^{k}(\Omega)$, and $\mathbf{C}_{0}^{\infty}(\Omega)$ are defined as usual, the superscript indicating continuous derivatives to a certain order and the subscript zero indicating functions with compact support.

The space $L^{P}(\Omega)$, the Hölder space $C^{k, \gamma}(\Omega)$, and the Sobolev space $W_{p}^{k}(\Omega)$ and their vector-valued analogues $\mathbf{L}^{P}(\Omega)$, 
$\mathbf{C}^{k, \gamma}(\Omega)$, and $\mathbf{W}_{p}^{k}(\Omega)$ are also defined as usual. In particular, $\mathbf{H}^{1}(\Omega)=\mathbf{W}_{2}^{1}(\Omega)$.

For functions depending on space and time, for a given space $\mathbf{V}$ of space-dependent functions, we define (for some $T>0)$

$$
\begin{gathered}
L^{2}(0, T ; \mathbf{V})=\{\mathbf{v}:(0, T) \longrightarrow \mathbf{V} \mid \mathbf{v} \text { is measurable and } \\
\left.\qquad \int_{0}^{T}\|\mathbf{v}(t)\|_{V}^{2} d t<\infty\right\}
\end{gathered}
$$

with norm $\|\mathbf{v}\|_{L^{2}(0, T ; V)}=\left(\int_{0}^{T}\|\mathbf{v}(t)\|_{V}^{2} d t\right)^{1 / 2}$.

When considering functions which depend only on time, we define the space $\mathbf{L}^{\infty}(0, T)=\left\{\mathbf{z}:(0, T) \rightarrow \mathbb{R}^{3}\right.$ । ess $\left.\sup _{t \in(0, T)}|\mathbf{z}(t)|<\infty\right\}$ endowed with the norm

$$
\|\mathbf{z}\|_{L^{\infty}(0, T)}=\operatorname{ess} \sup _{t \in(0, T)}|\mathbf{z}(t)| .
$$

1.2. Preliminaries. In this subsection, we recall some assumptions used in $[2,4]$, and we are going to make use of them throughout our analysis. In what follows, $\Omega$ is a bounded domain of $\mathbb{R}^{3}$ with boundary $\Gamma$ sufficiently smooth. $\Gamma$ consists of the artery wall denoted by $\Gamma_{\text {wall }}$ and some artificial sections. The velocity is required to be zero on the artery wall. To account for homogeneous Dirichlet boundary conditions on the artery wall, we define

$$
\mathbf{X} \equiv\left\{\boldsymbol{\varphi} \in \mathbf{H}^{1}(\Omega):\left.\varphi\right|_{\Gamma_{\text {wall }}}=0\right\} .
$$

Note that Poincaré's inequality

$$
\|\varphi\| \leq C_{\Omega}\|\nabla \varphi\|
$$

holds for $\varphi \in \mathbf{X}[2,4]$. The artificial sections consist of the upstream section on the side of the heart and the downstream sections on the side of the peripheral vessels. Rather than giving serious thought to the artificial sections boundary conditions, in seeking a variational formulation, the test space is left free on these portions of the boundary. Accordingly, we introduce

$$
\mathbf{V} \equiv\left\{\boldsymbol{\varphi} \in \mathbf{H}^{1}(\Omega):\left.\varphi\right|_{\Gamma_{\text {wall }}}=0, \nabla \cdot \boldsymbol{\varphi}=0\right\}
$$

as the test space. To prove an existence theorem for a NavierStokes problem, either steady or nonsteady, it is convenient to construct the solution as a limit of Galerkin approximations in terms of the eigenfunctions of the corresponding steady Stokes problem. This use of the Stokes eigenfunctions originated with Prodi and was further developed by Heywood [5]. To define the corresponding Stokes operator, we introduce $\mathbf{V}^{\star}$ as the completion of $\mathbf{V}$ with respect to the norm of $\mathbf{L}^{2}(\Omega)$. Then for every $\mathbf{f} \in \mathbf{V}^{\star}$, there exists exactly one $\mathbf{v} \in \mathbf{V}$ satisfying

$$
(\nabla \mathbf{v}, \nabla \varphi)=(\mathbf{f}, \varphi), \quad \forall \varphi \in \mathbf{V} .
$$

Moreover, for each $\mathbf{v} \in \mathbf{V}$, there exist at most one $\mathbf{f} \in \mathbf{V}^{\star}$ such that (6) holds. In this way, a one-to-one correspondence can be defined between elements $\mathbf{f}$ of $\mathbf{V}^{\star}$ and functions $\mathbf{v}$ belonging to an allowing suitable subspace of $\mathbf{V}$ that we denote by $D(\widetilde{\Delta})$. The Stokes operator

$$
\widetilde{\Delta}: D(\widetilde{\Delta}) \longrightarrow \mathbf{V}^{\star}
$$

is defined setting

$$
-\widetilde{\Delta} \mathbf{v}=\mathbf{f}
$$

so that (6) is satisfied.

The inverse operator $\widetilde{\Delta}^{-1}$ is completely continuous and self-adjoint. Therefore it possesses a sequence of eigenfunctions $\left\{\mathbf{a}_{k}\right\}_{k=1}^{\infty}$, which are complete and orthogonal in both $\mathbf{V}$ and $\mathbf{V}^{\star}$.

In what follows, $c_{i}(i=1,2, \ldots)$ will denote generic constants, not necessarily the same at different places. The inequalities

$$
\begin{gathered}
\sup _{\Omega}|\mathbf{v}| \leq c_{1}\|\nabla \mathbf{v}\|^{1 / 2}\|\widetilde{\Delta} \mathbf{v}\|^{1 / 2}, \\
\|\nabla \mathbf{v}\| \leq c_{2}\|\widetilde{\Delta} \mathbf{v}\|
\end{gathered}
$$

are satisfied for every $\mathbf{v} \in D(\widetilde{\Delta})$, provided that $\Omega$ is a bounded domain (see [4] page 178).

Next, we provide a few theorems $[1,3,6]$ used throughout our analysis.

Theorem 1 (Brouwer's fixed point theorem). Let $X$ be a finite dimensional linear space endowed with a norm. Assume

$$
u: B(0,1) \longrightarrow B(0,1)
$$

is continuous, where $B(0,1)$ denotes the closed-unit ball in $X$. Then $u$ has a fixed point; that is, there exists a point $x \in B(0,1)$ with

$$
u(x)=x .
$$

Theorem 2 (Rellich-Kondrachov compactness theorem). Assume $U$ is a bounded open subset of $\mathbb{R}^{n}$, with a $C^{1}$ boundary. Suppose $1 \leq p<n$. Then

$$
W_{p}^{1}(U) \subset \subset L^{q}(U)
$$

for each $1 \leq q<p^{\star}$, where $1 / p^{\star}=(1 / p)-(1 / n)$.

As a result of that, we have

$$
\|u\|_{L^{q}(U)} \leq C\|u\|_{W_{p}^{1}(U)} .
$$

Theorem 3 (trace inequality for solenoidal functions). Let $\mathbf{u}$ be a solenoidal function defined on $U$. Then the following inequality holds:

$$
\int_{\partial U}(\mathbf{u} \cdot \mathbf{n})^{2} d s \leq c\|\mathbf{u}\|^{2} .
$$

Theorem 4 (weak compactness). Let $X$ be a reflexive Banach space and suppose that the sequence $\left\{\mathbf{u}_{k}\right\}_{k=1}^{\infty} \subset X$ is bounded. Then there exists a subsequence $\left\{\mathbf{u}_{k j}\right\}_{j=1}^{\infty} \subset\left\{\mathbf{u}_{k}\right\}_{k=0}^{\infty}$ and $\mathbf{u} \in X$ such that

$$
\mathbf{u}_{k j} \rightarrow \mathbf{u} \text {. }
$$


In other words, bounded sequences in a reflexive Banach space are weakly precompact. In particular, a bounded sequence in a Hilbert space contains a weakly convergent subsequence.

\section{Formulation of the Problem}

Let $\Omega \subset \mathbb{R}^{3}$ be the artery portion where we aim at providing a detailed flow analysis. For each $\mathbf{x} \in \Omega$ and at any time $t>$ 0 , we denote by $\mathbf{u}(\mathbf{x}, t)$ and $p(\mathbf{x}, t)$ the blood velocity and pressure, respectively. In larger vessels, it is reasonable to assume that blood has a constant viscosity, because the vessel diameters are large compared with the individual cell diameters and because shear rates are high enough for viscosity to be independent of them. Hence, in these vessels the nonNewtonian behavior becomes insignificant and blood can be considered to be a Newtonian fluid ([4] and references therein). In what follows, we assume that the vessels are large enough; blood density and blood viscosity are assumed to be constant. Under these assumptions, blood flow can be described by the Navier-Stokes equations

$$
\begin{array}{r}
\rho \frac{\partial \mathbf{u}}{\partial t}+\rho(\mathbf{u} \cdot \nabla) \mathbf{u}+\nabla \cdot \mathbf{T}=0, \quad \nabla \cdot \mathbf{u}=0, \\
\mathbf{x} \in \Omega, t>0,
\end{array}
$$

where $\mathbf{T}=\mathbf{T}=p \mathbf{I}-\mu\left(\nabla \mathbf{u}+(\nabla \mathbf{u})^{T}\right)$. The equations are expressions of balance of linear momentum and incompressibility. We are neglecting the presence of any external forces. Then (18) is obtained by substitution of the divergence-free constraint into the expression for the stress in (17) as follows

$$
\begin{array}{r}
\rho \frac{\partial \mathbf{u}}{\partial t}+\rho(\mathbf{u} \cdot \nabla) \mathbf{u}-\mu \Delta \mathbf{u}+\nabla p=0, \quad \nabla \cdot \mathbf{u}=0, \\
\mathbf{x} \in \Omega, t>0 .
\end{array}
$$

In fact,

$$
\begin{aligned}
\nabla \cdot \mathbf{T} & =\nabla \cdot\left(p \mathbf{I}-\mu\left(\nabla \mathbf{u}+(\nabla \mathbf{u})^{T}\right)\right) \\
& =\nabla p-\mu \Delta \mathbf{u} \quad \text { provided that } \nabla \cdot \mathbf{u}=0 .
\end{aligned}
$$

For the sake of simplicity, we normalize $\rho$ to 1 .

2.1. Initial and Boundary Conditions. The initial condition requires the specification of the flow velocity at the initial time; for example, for $t_{0}=0$;

$$
\mathbf{u}(\mathbf{x}, 0)=\mathbf{u}_{0}(\mathbf{x}), \quad \mathbf{x} \in \Omega,
$$

where the given initial velocity field $\mathbf{u}_{0}$ is divergence-free. The system (18) has to be provided with boundary conditions. In this respect, we split the boundary $\Gamma$ into different parts. In the present work, we are assuming that the wall is rigid so that no-slip boundary condition

$$
\mathbf{u}(\mathbf{x}, t)=0, \quad \mathbf{x} \in \Gamma_{\text {wall }}, t>0,
$$

holds. The other parts of $\Gamma$ are the artificial boundaries which bound the computational domain. For the sake of clarity, we distinguish the upstream section on the side of the heart denoted by $\Gamma_{1}$ and the downstream sections on the side of the peripheral vessels denoted by $\Gamma_{2}$ and $\Gamma_{3}$. As introduced in [4], the following boundary conditions are provided:

$$
\begin{array}{r}
p \mathbf{n}-\mu(\nabla \mathbf{u}) \mathbf{n}-K_{i}(\mathbf{u} \cdot \mathbf{n}) \mathbf{n}=P_{i} \mathbf{n} \\
\text { for } t>0, \mathbf{x} \in \Gamma_{i}(i=1,2,3),
\end{array}
$$

where $K_{i}$ is a suitable nonnegative constant, $P_{i}=P_{i}(t)$ is the prescribed mean pressure on each artificial section $\Gamma_{i}$, and n represents the outward normal unit vector on every part of the vessel boundary. These conditions can be considered as a generalization of the mean pressure drop problem investigated in [2] in the sense that when $K_{i}=0(i=1,2$, 3 ), we recover the usual Neumann or natural conditions associated with (18). The physical justification for the case in which $K_{i} \neq 0$ is provided in ([4] Figure 4.1).

The mathematical formulation of the problem is therefore described by the system of differential equations:

$$
\begin{gathered}
\frac{\partial \mathbf{u}}{\partial t}+(\mathbf{u} \cdot \nabla) \mathbf{u}-\mu \Delta \mathbf{u}+\nabla p=0, \quad \mathbf{x} \in \Omega, t>0, \\
\nabla \cdot \mathbf{u}=0, \quad \mathbf{x} \in \Omega, t>0, \\
\mathbf{u}(\mathbf{x}, 0)=\mathbf{u}_{0}(\mathbf{x}), \quad \mathbf{x} \in \Omega, \\
\mathbf{u}(\mathbf{x}, t)=0, \quad \mathbf{x} \in \Gamma_{\text {wall }}, t>0, \\
p \mathbf{n}-\mu(\nabla \mathbf{u}) \mathbf{n}-K_{i}(\mathbf{u} \cdot \mathbf{n}) \mathbf{n}=P_{i} \mathbf{n}, \quad \text { for } t>0, \mathbf{x} \in \Gamma_{i} .
\end{gathered}
$$

Next we define the following bilinear and trilinear forms:

$$
\begin{aligned}
& \begin{array}{l}
a: \mathbf{H}^{1}(\Omega) \times \mathbf{H}^{1}(\Omega) \rightarrow \mathbb{R} \text { such that } \\
\begin{aligned}
a\left(\mathbf{v}^{(1)}, \mathbf{v}^{(2)}\right)= & \mu\left(\nabla \mathbf{v}^{(1)}, \nabla \mathbf{v}^{(2)}\right) \\
& \quad+\sum_{i=1}^{3} K_{i} \int_{\Gamma_{i}}\left(\mathbf{v}^{(1)} \cdot \mathbf{n}\right) \mathbf{n} \cdot \mathbf{v}^{(2)} d s,
\end{aligned} \\
b: \mathbf{H}^{1}(\Omega) \times \mathbf{H}^{1}(\Omega) \times \mathbf{H}^{1}(\Omega) \rightarrow \mathbb{R} \text { such that } \\
\quad b\left(\mathbf{v}^{(1)}, \mathbf{v}^{(2)}, \mathbf{v}^{(3)}\right)=\left(\left(\mathbf{v}^{(1)} \cdot \nabla\right) \mathbf{v}^{(2)}, \mathbf{v}^{(3)}\right) .
\end{array}
\end{aligned}
$$

Following [5] we set

$$
c(t, \mathbf{v})=-\sum_{i=1}^{3} P_{i}(t) \int_{\Gamma_{i}} \mathbf{n} \cdot \mathbf{v} d s, \quad \text { for each } \mathbf{v} \in \mathbf{H}^{1}(\Omega) .
$$

Furthermore, if we set

$$
\widetilde{P}=\sup _{t \geq 0} \sum_{i=1}^{3}\left|P_{i}\right|, \quad K=\max _{1 \leq i \leq 3} K_{i}
$$

and consider the sequence $\left\{\mathbf{a}_{k}\right\}_{k=1}^{\infty}$ of eigenfunctions of $\widetilde{\Delta}^{-1}$, the following estimates hold for every $\mathbf{v} \in \operatorname{span}\left\{\mathbf{a}_{k}, k=\right.$ $1,2, \ldots\}$ :

$$
\left|\sum_{i=1}^{3} P_{i} \int_{\Gamma_{i}} \widetilde{\Delta} \mathbf{v} \cdot \mathbf{n} d s\right| \leq c_{3} \widetilde{P}\|\widetilde{\Delta} \mathbf{v}\|
$$


where $c_{3}$ depends on the trace inequality (15) and

$$
\left|\sum_{i=1}^{3} K_{i} \int_{\Gamma_{i}}(\mathbf{v} \cdot \mathbf{n}) \mathbf{n} \cdot \widetilde{\Delta} \mathbf{v} d s\right| \leq c_{4} K\|\nabla \mathbf{v}\|\|\widetilde{\Delta} \mathbf{v}\|,
$$

where $c_{4}$ depends on the trace inequality (15) and Poincaré's inequality [3].

The former is a consequence of the trace inequality for solenoidal functions [2] and the proof of (29) is given in [4]

\section{The Stationary Problem}

Our purpose is to establish the existence of a weak solution to the stationary version of problem (23). We are going to consider the inner product with $\varphi \in \mathbf{V}$ in order to formulate the weak formulation.

3.1. Weak Formulation. Assume that $\mathbf{u}$ is a solution of the boundary value problem described above and $\varphi$ is a smooth solenoidal vector-valued function defined on $\Omega$. The function u satisfies the following identities:

$$
\begin{aligned}
&((\mathbf{u} \cdot \nabla) \mathbf{u}-\mu \Delta \mathbf{u}+\nabla p, \varphi)=0, \mathbf{x} \in \Omega, \\
&\left(p \mathbf{n}-\mu(\nabla \mathbf{u}) \mathbf{n}-K_{i}(\mathbf{u} \cdot \mathbf{n}) \mathbf{n}, \boldsymbol{\varphi}\right)=\left(P_{i} \mathbf{n}, \boldsymbol{\varphi}\right), \\
& \mathbf{x} \in \Gamma_{i}, \quad i=1,2,3 .
\end{aligned}
$$

We notice that $\left.\varphi\right|_{\Gamma_{\text {wall }}}=0$ since $\varphi \in \mathrm{V}$.

We calculate

$$
\begin{aligned}
(\nabla p, \boldsymbol{\varphi}) & =-\int_{\Omega} p(\nabla \cdot \boldsymbol{\varphi}) d \mathbf{x}+\int_{\Gamma} p(\boldsymbol{\varphi} \cdot \mathbf{n}) d s \\
& =\sum_{i=1}^{3} \int_{\Gamma_{i}}\left(P_{i} \mathbf{n}+\mu(\nabla \mathbf{u}) \mathbf{n}+K_{i}(\mathbf{u} \cdot \mathbf{n}) \mathbf{n}\right) \cdot \boldsymbol{\varphi} d s \\
(\Delta \mathbf{u}, \boldsymbol{\varphi}) & =-\int_{\Omega} \sum_{i, j=1}^{3} \frac{\partial \varphi_{i}}{\partial x_{j}} \frac{\partial u_{i}}{\partial x_{j}} d \mathbf{x}+\int_{\Gamma} \sum_{i=1}^{3} \varphi_{i} \sum_{j=1}^{3} \frac{\partial u_{i}}{\partial x_{j}} n_{j} d s \\
& =-(\nabla \boldsymbol{\varphi}, \nabla \mathbf{u})+\sum_{i=1}^{3} \int_{\Gamma_{i}}(\mathbf{n} \cdot \nabla) \mathbf{u} \cdot \boldsymbol{\varphi} d s
\end{aligned}
$$

to obtain

$$
\begin{aligned}
((\mathbf{u} \cdot \nabla) \mathbf{u}, \boldsymbol{\varphi})+\mu(\nabla \mathbf{u}, \nabla \boldsymbol{\varphi})= & -\sum_{i=1}^{3} \int_{\Gamma_{i}} K_{i}(\mathbf{u} \cdot \mathbf{n}) \mathbf{n} \cdot \boldsymbol{\varphi} d s \\
& -\sum_{i=1}^{3} P_{i} \int_{\Gamma_{i}} \mathbf{n} \cdot \boldsymbol{\varphi} d s .
\end{aligned}
$$

It is clear that (33) does not depend on the pressure $p$; therefore, the weak formulation of the problem reads as follows.
Given a divergence free velocity field $\mathbf{u}_{0} \in \mathbf{V}$ and nonnegative constants $P_{i}$ and $K_{i}(i=1,2,3)$, find $\mathbf{u} \in \mathbf{V}$ such that

$$
\begin{aligned}
((\mathbf{u} \cdot \nabla) \mathbf{u}, \boldsymbol{\varphi})+\mu(\nabla \mathbf{u}, \nabla \boldsymbol{\varphi})= & -\sum_{i=1}^{3} \int_{\Gamma_{i}} K_{i}(\mathbf{u} \cdot \mathbf{n}) \mathbf{n} \cdot \boldsymbol{\varphi} d s \\
& -\sum_{i=1}^{3} P_{i} \int_{\Gamma_{i}} \mathbf{n} \cdot \boldsymbol{\varphi} d s
\end{aligned}
$$

holds for all $\varphi \in V$, where $\mathbf{V}$, defined in (5), is the space of test functions.

3.2. Galerkin Approximations. The inverse operator $\widetilde{\Delta}^{-1}$ of the Stokes operator $\widetilde{\Delta}$ is self-adjoint and possesses a sequence of eigenfunctions $\left\{\mathbf{a}_{k}\right\}$ which are orthogonal in V. Following [6] we fix a positive integer $n$. Galerkin approximations

$$
\mathbf{u}_{n}=\sum_{k=1}^{n} d_{n}^{k} \mathbf{a}_{k}
$$

are defined as solutions of the finite system of equations $(k=$ $1, \ldots, n)$ as follows:

$$
\begin{aligned}
\left(\left(\mathbf{u}_{n} \cdot \nabla\right) \mathbf{u}_{n}, \mathbf{a}_{k}\right)+\mu\left(\nabla \mathbf{u}_{n}, \nabla \mathbf{a}_{k}\right) & \\
= & -\sum_{i=1}^{3} \int_{\Gamma_{i}} K_{i}\left(\mathbf{u}_{n} \cdot \mathbf{n}\right) \mathbf{n} \cdot \mathbf{a}_{k} d s \\
& -\sum_{i=1}^{3} P_{i} \int_{\Gamma_{i}} \mathbf{n} \cdot \mathbf{a}_{k} d s .
\end{aligned}
$$

This is a system of linear equations for constant unknowns $d_{n}^{k}(k=1, \ldots, n)$. The identity (37) for $\mathbf{u}_{n}$ is obtained by multiplying (36) through by $d_{n}^{k}$ and summing over $k=1$, $\ldots, n$ :

$$
\begin{aligned}
\left(\left(\mathbf{u}_{n} \cdot \nabla\right) \mathbf{u}_{n}, \mathbf{u}_{n}\right)+\mu\left(\nabla \mathbf{u}_{n}, \nabla \mathbf{u}_{n}\right) & =-\sum_{i=1}^{3} \int_{\Gamma_{i}} K_{i}\left(\mathbf{u}_{n} \cdot \mathbf{n}\right) \mathbf{n} \cdot \mathbf{u}_{n} d s \\
& -\sum_{i=1}^{3} P_{i} \int_{\Gamma_{i}} \mathbf{n} \cdot \mathbf{u}_{n} d s .
\end{aligned}
$$

This implies that

$$
\begin{aligned}
\mu\left\|\nabla \mathbf{u}_{n}\right\|^{2} \leq & \left|\left(\left(\mathbf{u}_{n} \cdot \nabla\right) \mathbf{u}_{n}, \mathbf{u}_{n}\right)\right| \\
& +\left|\sum_{i=1}^{3} \int_{\Gamma_{i}} K_{i}\left(\mathbf{u}_{n} \cdot \mathbf{n}\right) \mathbf{n} \cdot \mathbf{u}_{n} d s\right| \\
& +\left|\sum_{i=1}^{3} P_{i} \int_{\Gamma_{i}} \mathbf{n} \cdot \mathbf{u}_{n} d s\right| .
\end{aligned}
$$

Together with (4), we make use of (28) to obtain

$$
\left|\sum_{i=1}^{3} P_{i}(t) \int_{\Gamma_{i}} \mathbf{n} \cdot\left(\mathbf{u}_{n}\right) d s\right| \leq c_{3} \widetilde{P}\left\|\mathbf{u}_{n}\right\| \leq c_{5} \widetilde{P}\left\|\nabla \mathbf{u}_{n}\right\| .
$$


Likewise, from (29), we have

$$
\left|\sum_{i=1}^{3} K_{i} \int_{\Gamma_{i}}\left(\mathbf{u}_{n} \cdot \mathbf{n}\right) \mathbf{n} \cdot \mathbf{u}_{n} d s\right| \leq c_{4} K\left\|\nabla \mathbf{u}_{n}\right\|\left\|\mathbf{u}_{n}\right\| \leq c_{6} K\left\|\nabla \mathbf{u}_{n}\right\|^{2} .
$$

Furthermore, making use of Hölder's inequality ([6], page 623), one obtains

$$
\begin{aligned}
\left(\left(\mathbf{u}_{n} \cdot \nabla\right) \mathbf{u}_{n}, \mathbf{u}_{n}\right) & \leq\left\|\mathbf{u}_{n}\right\|_{L^{6}}\left\|\nabla \mathbf{u}_{n}\right\|\left\|\mathbf{u}_{n}\right\|_{L^{3}} \\
& \leq c_{7}\left\|\nabla \mathbf{u}_{n}\right\|^{3},
\end{aligned}
$$

where $c_{7}$ depends on Rellich-Kondrachov compactness inequality [1], Poincaré's inequality [3], and Sobolev inequality ([6], page 270). We make use of these inequalities in (38) and we obtain

$$
\mu\left\|\nabla \mathbf{u}_{n}\right\|^{2} \leq c_{7}\left\|\nabla \mathbf{u}_{n}\right\|^{3}+c_{5} \widetilde{P}\left\|\nabla \mathbf{u}_{n}\right\|+c_{6} K\left\|\nabla \mathbf{u}_{n}\right\|^{2}
$$

Set $\xi:=\mu-c_{6} K$. It follows that

$$
\xi\left\|\nabla \mathbf{u}_{n}\right\| \leq c_{7}\left\|\nabla \mathbf{u}_{n}\right\|^{2}+c_{5} \widetilde{P}
$$

where $\widetilde{P}=\sum_{i=1}^{3}\left|P_{i}\right|, K=\max _{1 \leq i \leq 3} K_{i}$.

Theorem 5. Assume

$$
K \leq \frac{\mu}{c_{6}}, \quad \widetilde{P} \leq \frac{\xi^{2}}{4 c_{5} c_{7}} .
$$

For each integer $n=1,2, \ldots$, there exists a function $\mathbf{u}_{n}$ of the form (35) satisfying (36) such that

$$
\left\|\nabla \mathbf{u}_{n}\right\| \leq \frac{\xi}{2 c_{7}}\left[1-\sqrt{\left(1-\frac{4 c_{5} c_{7} \widetilde{P}}{\xi^{2}}\right)}\right] .
$$

Proof. Owing to Poincarés inequality (4), $\|\nabla \varphi\|$ is a norm equivalent to $\|\varphi\|_{1}$ for all $\varphi \in \mathbf{V}$; therefore, (45) defines a closed ball in $\operatorname{span}\left\{\mathbf{a}_{1}, \ldots, \mathbf{a}_{n}\right\}$. To prove the solvability of the finite-dimensional problem (36), we follow Fujita in using Brouwer's fixed point theorem (see Theorem 1.1 in [7]), applying it to the continuous mapping $\mathbf{v} \rightarrow \mathbf{w}$ defined by the linear problem $(k=1, \ldots, n)$ :

$$
\begin{aligned}
\left((\mathbf{v} \cdot \nabla) \mathbf{w}, \mathbf{a}_{k}\right)+\mu\left(\nabla \mathbf{w}, \nabla \mathbf{a}_{k}\right)= & -\sum_{i=1}^{3} \int_{\Gamma_{i}} K_{i}(\mathbf{w} \cdot \mathbf{n}) \mathbf{n} \cdot \mathbf{a}_{k} d s \\
& -\sum_{i=1}^{3} P_{i} \int_{\Gamma_{i}} \mathbf{n} \cdot \mathbf{a}_{k} d s .
\end{aligned}
$$

Equation (46) is a system of $n$ linear equations. These linear equations are uniquely solvable if $\mathbf{v}$ lies in the ball defined by (45), because then $\mathbf{w}=0$ is the only solution of the corresponding homogeneous equation $\left(K_{i}=P_{i}=0\right.$, $1 \leq i \leq 3$ ). In fact, if $\mathbf{v}$ satisfies (45) and $\mathbf{w}$ satisfies (46) with $K_{i}=P_{i}=0$, we have

$$
\begin{aligned}
\xi\|\nabla \mathbf{w}\|^{2} & \leq c_{7}\|\nabla \mathbf{v}\|\|\nabla \mathbf{w}\|^{2} \\
& \leq c_{7} \frac{\xi}{2 c_{7}}\left[1-\sqrt{\left(1-\frac{4 c_{5} c_{7} \widetilde{P}}{\xi^{2}}\right)}\right]\|\nabla \mathbf{w}\|^{2} \\
& \leq \frac{\xi}{2}\|\nabla \mathbf{w}\|^{2} .
\end{aligned}
$$

Together with Poincaré's inequality (4), this imply that $\mathbf{w}=0$. To see that the mapping $\mathbf{v} \rightarrow \mathbf{w}$ takes the ball defined by (45) into itself, suppose that $\mathbf{v}$ satisfies (45). Then, similarly to (43), we obtain that

$$
\xi\|\nabla \mathbf{w}\| \leq c_{7}\|\nabla \mathbf{v}\|\|\nabla \mathbf{w}\|+c_{5} \widetilde{P},
$$

and therefore,

$$
\|\nabla \mathbf{w}\| \leq \frac{c_{5} \widetilde{P}}{\xi-c_{7}\|\nabla \mathbf{v}\|}
$$

$$
\begin{aligned}
& \leq \frac{c_{5} \widetilde{P}}{\xi-(\xi / 2)\left[1-\sqrt{\left(1-\left(4 c_{5} c_{7} \widetilde{P} / \xi^{2}\right)\right)}\right.} \\
& \leq \frac{\xi}{2 c_{7}}\left[1-\sqrt{\left(1-\frac{4 c_{5} c_{7} \widetilde{P}}{\xi^{2}}\right)}\right] .
\end{aligned}
$$

Thus, (46) defines a continuous mapping $\mathbf{v} \rightarrow \mathbf{w}$ of the closed ball

$$
\left\{\boldsymbol{\varphi} \in \operatorname{span}\left\{\mathbf{a}_{1}, \ldots, \mathbf{a}_{n}\right\}:\|\nabla \boldsymbol{\varphi}\| \leq \frac{\xi}{2 c_{7}}\left[1-\sqrt{\left(1-\frac{4 c_{5} c_{7} \widetilde{P}}{\xi^{2}}\right)}\right]\right\}
$$

into itself. The map has at least one fixed point, and any such fixed point is a solution of (36). $\mathbf{u}_{n}$ is chosen to be any one of these fixed points. Hence, Brouwer's fixed point has been applied and has given the existence of Galerkin approximations

$$
\mathbf{u}_{n}=\sum_{k=1}^{n} d_{n}^{k} \mathbf{a}_{k}
$$

satisfying

$$
\left\|\nabla \mathbf{u}_{n}\right\| \leq \frac{\xi}{2 c_{7}}\left[1-\sqrt{\left(1-\frac{4 c_{5} c_{7} \widetilde{P}}{\xi^{2}}\right)}\right] .
$$

\subsection{Existence of a Weak Solution}

Theorem 6. Assume

$$
K \leq \frac{\mu}{c_{6}}, \quad \widetilde{P} \leq \frac{\xi^{2}}{4 c_{5} c_{7}} .
$$

There exists a weak solution to the stationary version of problem (23). 
Proof. By Poincarés inequality [3], the fact that the sequence $\left\{\left\|\nabla \mathbf{u}_{n}\right\|\right\}_{n=1}^{\infty}$ is bounded implies that the sequence $\left\{\mathbf{u}_{n}\right\}_{n=1}^{\infty}$ is bounded in $\mathbf{V}$. As a result, Theorem 4 yields the existence of a subsequence $\left\{\mathbf{u}_{n_{q}}\right\}_{q=1}^{\infty} \subset\left\{\mathbf{u}_{n}\right\}_{n=1}^{\infty}$ such that

$$
\mathbf{u}_{n_{q}} \rightarrow \mathbf{u} \text { weakly in } \mathbf{V} .
$$

Next we show that the weak limit $\mathbf{u}$ is in fact a weak solution. In this respect, we are going to show that

$$
\begin{aligned}
((\mathbf{u} \cdot \nabla) \mathbf{u}, \boldsymbol{\varphi})+\mu(\nabla \mathbf{u}, \nabla \boldsymbol{\varphi})= & -\sum_{i=1}^{3} \int_{\Gamma_{i}} K_{i}(\mathbf{u} \cdot \mathbf{n}) \mathbf{n} \cdot \boldsymbol{\varphi} d s \\
& -\sum_{i=1}^{3} P_{i} \int_{\Gamma_{i}} \mathbf{n} \cdot \boldsymbol{\varphi} d s,
\end{aligned}
$$

for each $\varphi \in \mathbf{V}$.

Fix an integer $k,(k=1,2, \ldots)$. From (36), we have the identity

$$
\begin{aligned}
\left(\left(\mathbf{u}_{n} \cdot \nabla\right) \mathbf{u}_{n}, \mathbf{a}_{k}\right)+\mu\left(\nabla \mathbf{u}_{n}, \nabla \mathbf{a}_{k}\right) & \\
= & -\sum_{i=1}^{3} \int_{\Gamma_{i}} K_{i}\left(\mathbf{u}_{n} \cdot \mathbf{n}\right) \mathbf{n} \cdot \mathbf{a}_{k} d s \\
& -\sum_{i=1}^{3} P_{i} \int_{\Gamma_{i}} \mathbf{n} \cdot \mathbf{a}_{k} d s .
\end{aligned}
$$

We recall (54), to find upon passing to weak limits that

$$
\begin{aligned}
\left((\mathbf{u} \cdot \nabla) \mathbf{u}, \mathbf{a}_{k}\right)+\mu\left(\nabla \mathbf{u}, \nabla \mathbf{a}_{k}\right)= & -\sum_{i=1}^{3} \int_{\Gamma_{i}} K_{i}(\mathbf{u} \cdot \mathbf{n}) \mathbf{n} \cdot \mathbf{a}_{k} d s \\
& -\sum_{i=1}^{3} P_{i} \int_{\Gamma_{i}} \mathbf{n} \cdot \mathbf{a}_{k} d s .
\end{aligned}
$$

Since $\left\{\mathbf{a}_{k}\right\}_{k=1}^{\infty}$ is a basis of $\mathbf{V}$, it follows that

$$
\begin{aligned}
((\mathbf{u} \cdot \nabla) \mathbf{u}, \boldsymbol{\varphi})+\mu(\nabla \mathbf{u}, \nabla \boldsymbol{\varphi})= & -\sum_{i=1}^{3} \int_{\Gamma_{i}} K_{i}(\mathbf{u} \cdot \mathbf{n}) \mathbf{n} \cdot \boldsymbol{\varphi} d s \\
& -\sum_{i=1}^{3} P_{i} \int_{\Gamma_{i}} \mathbf{n} \cdot \boldsymbol{\varphi} d s,
\end{aligned}
$$

for each $\varphi \in V$.

Note that, in taking this limit, there is no difficulty with the nonlinear term. In fact, we have that

$$
\begin{aligned}
\left(\mathbf{u}_{n_{q}} \cdot \nabla\right) \mathbf{u}_{n_{q}}-(\mathbf{u} \cdot \nabla) \mathbf{u}= & \left(\mathbf{u}_{n_{q}} \cdot \nabla\right) \mathbf{u}_{n_{q}}+\left(\mathbf{u}_{n_{q}} \cdot \nabla\right) \mathbf{u} \\
& -\left(\mathbf{u}_{n_{q}} \cdot \nabla\right) \mathbf{u}-(\mathbf{u} \cdot \nabla) \mathbf{u} \\
= & \left(\mathbf{u}_{n_{q}} \cdot \nabla\right)\left(\mathbf{u}_{n_{q}}-\mathbf{u}\right) \\
& +\left(\left(\mathbf{u}_{n_{q}}-\mathbf{u}\right) \cdot \nabla\right) \mathbf{u} .
\end{aligned}
$$

Also, $\mathbf{a}_{k} \in \mathbf{H}^{2}(\Omega)$ is an eigenfunction of the inverse of the stokes operator $\widetilde{\Delta}$ and $\mathbf{H}^{2}(\Omega) \subset \mathbf{C}(\bar{\Omega})$. This implies that $\mathbf{a}_{k} \in$ $\mathrm{C}(\bar{\Omega})[6]$. It follows that

$$
\begin{aligned}
& \left(\left(\mathbf{u}_{n_{q}} \cdot \nabla\right) \mathbf{u}_{n_{q}}-(\mathbf{u} \cdot \nabla) \mathbf{u}, \mathbf{a}_{k}\right) \\
& =\left(\left(\mathbf{u}_{n_{q}} \cdot \nabla\right)\left(\mathbf{u}_{n_{q}}-\mathbf{u}\right), \mathbf{a}_{k}\right) \\
& \quad+\left(\left(\left(\mathbf{u}_{n_{q}}-\mathbf{u}\right) \cdot \nabla\right) \mathbf{u}, \mathbf{a}_{k}\right) \longrightarrow 0
\end{aligned}
$$

(see [5], page 651).

\section{The Nonstationary Problem}

The mathematical analysis of the Navier-Stokes problem is based on its weak formulation.

4.1. Weak Formulation. Assume that $\mathbf{u}$ is a solution to problem (23) and $\varphi$ is a smooth solenoidal vector-valued function defined on $\Omega$. $\mathbf{u}$ satisfies the following identities:

$$
\begin{gathered}
\left(\frac{\partial \mathbf{u}}{\partial t}+(\mathbf{u} \cdot \nabla) \mathbf{u}-\mu \Delta \mathbf{u}+\nabla p, \boldsymbol{\varphi}\right)=0, \quad \mathbf{x} \in \Omega, t>0 \\
(\mathbf{u}(0), \boldsymbol{\varphi})=\left(\mathbf{u}_{0}, \boldsymbol{\varphi}\right), \quad \mathbf{x} \in \Omega \\
\left(p \mathbf{n}-\mu(\nabla \mathbf{u}) \mathbf{n}-K_{i}(\mathbf{u} \cdot \mathbf{n}) \mathbf{n}, \boldsymbol{\varphi}\right)=\left(P_{i} \mathbf{n}, \boldsymbol{\varphi}\right), \\
\mathbf{x} \in \Gamma_{i}, \quad i=1,2,3
\end{gathered}
$$

We calculate

$$
\begin{aligned}
(\nabla p, \boldsymbol{\varphi})= & \sum_{i=1}^{3} \int_{\Gamma_{i}}\left(P_{i} \mathbf{n}\right) \cdot \boldsymbol{\varphi} d s+\mu \sum_{i=1}^{3} \int_{\Gamma_{i}}(\mathbf{n} \cdot \nabla) \mathbf{u} \cdot \boldsymbol{\varphi} d s \\
& +\sum_{i=1}^{3} \int_{\Gamma_{i}} K_{i}(\mathbf{u} \cdot \mathbf{n}) \mathbf{n} \cdot \boldsymbol{\varphi} d s, \\
(\Delta \mathbf{u}, \boldsymbol{\varphi}) & =-(\nabla \boldsymbol{\varphi}, \nabla \mathbf{u})+\sum_{i=1}^{3} \int_{\Gamma_{i}}(\mathbf{n} \cdot \nabla) \mathbf{u} \cdot \boldsymbol{\varphi} d s,
\end{aligned}
$$

to obtain

$$
\left(\frac{\partial \mathbf{u}}{\partial t}, \boldsymbol{\varphi}\right)+b(\mathbf{u}, \mathbf{u}, \boldsymbol{\varphi})+a(\mathbf{u}, \boldsymbol{\varphi})=c(t, \boldsymbol{\varphi}),
$$

where $a, b$, and $c$ are defined by (24), (25), and (26), respectively. We notice that (63) does not depend on the pressure $p$. Therefore, the weak formulation of the problem reads as follows.

Given a divergence free velocity field $\mathbf{u}_{0} \in \mathbf{V}, P_{i} \in L^{\infty}(0$, $T$ ), and a nonnegative constant $K_{i}$ for $i=1,2,3$, find $\mathbf{u} \epsilon$ $L^{2}(0, T ; \mathbf{V})$ such that for all $t \geq 0$,

$$
\begin{aligned}
& \left(\frac{\partial \mathbf{u}}{\partial t}, \varphi\right)+b(\mathbf{u}, \mathbf{u}, \boldsymbol{\varphi})+a(\mathbf{u}, \varphi)=c(t, \varphi), \\
& (\mathbf{u}(0), \varphi)=\left(\mathbf{u}_{0}, \varphi\right), \quad \forall \varphi \in \mathbf{V},
\end{aligned}
$$

where $\mathbf{V}$, defined in (5), is the space of test functions. 
4.2. Galerkin Approximations. We are going to follow the same approach as in [6]. We will construct the weak solution of the initial boundary value problem by first solving for a finite dimensional approximation. We recall that the inverse operator $\widetilde{\Delta}^{-1}$ of the Stokes operator $\widetilde{\Delta}$ is self-adjoint and possesses a sequence of eigenfunctions $\left\{\mathbf{a}_{k}\right\}$ which are orthogonal in $\mathbf{V}$ and orthonormal in $\mathbf{L}^{2}(\Omega)$. Fix a positive integer $n$ and write

$$
\mathbf{u}_{n}(t):=\sum_{k=1}^{n} d_{n}^{k}(t) \mathbf{a}_{k}
$$

where we intend to select the coefficients $d_{n}^{k}(t)(0 \leq t \leq T$, $k=1, \ldots, n)$ to satisfy

$$
\begin{gathered}
d_{n}^{k}(0)=\left(\mathbf{u}_{0}, \mathbf{a}_{k}\right) \\
\left(\frac{\partial \mathbf{u}_{n}}{\partial t}, \mathbf{a}_{k}\right)+a\left(\mathbf{u}_{n}, \mathbf{a}_{k}\right)+b\left(\mathbf{u}_{n}, \mathbf{u}_{n}, \mathbf{a}_{k}\right)=c\left(t, \mathbf{a}_{k}\right)
\end{gathered}
$$

Theorem 7. For each integer $n=1,2, \ldots$, there exists a unique function $\mathbf{u}_{n}$ of the form (65) satisfying (66)-(67).

Proof. Assuming that $\mathbf{u}_{n}$ is given by (65), we observe using the fact that $\left\{\mathbf{a}_{k}\right\}_{k=1}^{\infty}$ is an orthonormal basis of $\mathbf{L}^{2}(\Omega)$ that

$$
\left(\frac{\partial \mathbf{u}_{n}}{\partial t}, \mathbf{a}_{k}\right)=\left(d_{n}^{k}\right)^{\prime}(t)
$$

where $\left(d_{n}^{k}\right)^{\prime}(t)$ is the derivative of $d_{n}^{k}(t)$ with respect to $t$.

Furthermore, from (24) and the fact that $a$ is a bilinear form, we have that

$$
a\left(\mathbf{u}_{n}, \mathbf{a}_{k}\right)=\sum_{i=1}^{n} A_{i}^{k} d_{n}^{i}(t),
$$

where $A_{i}^{k}=a\left(\mathbf{a}_{i}, \mathbf{a}_{k}\right)$.

From (25) and recalling that $b$ is a trilinear form, we have that

$$
b\left(\mathbf{u}_{n}, \mathbf{u}_{n}, \mathbf{a}_{k}\right)=\sum_{i, j=1}^{n} B_{i, j}^{k} d_{n}^{i}(t) d_{n}^{j}(t)
$$

where $B_{i, j}^{k}=b\left(\mathbf{a}_{i}, \mathbf{a}_{j}, \mathbf{a}_{k}\right)$.

Moreover, we set

$$
f^{k}(t):=c\left(t, \mathbf{a}_{k}\right) .
$$

It follows that (67) becomes the system of ODEs

$$
\begin{gathered}
d_{n}^{k^{\prime}}(t)+\sum_{i=1}^{n} A_{i}^{k} d_{n}^{i}(t)+\sum_{i, j=1}^{n} B_{i, j}^{k} d_{n}^{i}(t) d_{n}^{j}(t) \\
=f^{k}(t), \quad(k=1, \ldots, n),
\end{gathered}
$$

subject to the initial condition (66). According to standard existence theory for ordinary differential equations, there exists a unique function $\mathbf{d}_{n}(t)=\left(d_{n}^{1}(t), \ldots, d_{n}^{n}(t)\right)$ satisfying (66) and (72) for a.e. $0 \leq t \leq T$. And then $\mathbf{u}_{n}$ defined by (65) solves (67) for a.e. $0 \leq t \leq T$.
4.3. Energy Estimate. Our plan hereafter is to let $n \rightarrow \infty$. Before that, we will need some estimates, uniform in $n$.

Theorem 8. For each approximate solution $\mathbf{u}_{n}$, the following energy estimates hold:

$$
\begin{aligned}
\frac{d}{d t} & \left\|\nabla \mathbf{u}_{n}\right\|^{2}+\left(\mu-2 K c_{2} c_{4}\right)\left\|\widetilde{\Delta} \mathbf{u}_{n}\right\|^{2} \\
& \leq \frac{16 c_{1}^{4}}{\mu^{3}}\left\|\nabla \mathbf{u}_{n}\right\|^{6}+\frac{2 c_{3}^{2} \widetilde{P}^{2}}{\mu} .
\end{aligned}
$$

Proof. We denote by $\lambda_{k}$ the eigenvalue associated with the eigenfunction $\mathbf{a}_{k}$. Multiplying (63) by $-\lambda_{k} d_{n}^{k}$ and summing over $k=1, \ldots, n$, one obtains

$$
\left(\frac{\partial \mathbf{u}_{n}}{\partial t},-\widetilde{\Delta} \mathbf{u}_{n}\right)+b\left(\mathbf{u}_{n}, \mathbf{u}_{n},-\widetilde{\Delta} \mathbf{u}_{n}\right)+a\left(\mathbf{u}_{n},-\widetilde{\Delta} \mathbf{u}_{n}\right)=c\left(t,-\widetilde{\Delta} \mathbf{u}_{n}\right) .
$$

Now,

$$
\left(\frac{\partial \mathbf{u}_{n}}{\partial t},-\widetilde{\Delta} \mathbf{u}_{n}\right)=\left(\nabla\left(\frac{\partial \mathbf{u}_{n}}{\partial t}\right), \nabla \mathbf{u}_{n}\right)=\frac{1}{2} \frac{d}{d t}\left\|\nabla \mathbf{u}_{n}\right\|^{2},
$$

and also

$$
\begin{aligned}
& a\left(\mathbf{u}_{n},-\widetilde{\Delta} \mathbf{u}_{n}\right)= \mu\left(\nabla \mathbf{u}_{n}, \nabla\left(-\widetilde{\Delta} \mathbf{u}_{n}\right)\right) \\
&+\sum_{i=1}^{3} K_{i} \int_{\Gamma_{i}}\left(\mathbf{u}_{n} \cdot \mathbf{n}\right) \mathbf{n} \cdot\left(-\widetilde{\Delta} \mathbf{u}_{n}\right) d s \\
&= \mu\left\|\widetilde{\Delta} \mathbf{u}_{n}\right\|^{2}-\sum_{i=1}^{3} K_{i} \int_{\Gamma_{i}}\left(\mathbf{u}_{n} \cdot \mathbf{n}\right) \mathbf{n} \cdot\left(\widetilde{\Delta} \mathbf{u}_{n}\right) d s \\
& b\left(\mathbf{u}_{n}, \mathbf{u}_{n},-\widetilde{\Delta} \mathbf{u}_{n}\right)=-\left(\left(\mathbf{u}_{n} \cdot \nabla\right) \mathbf{u}_{n}, \widetilde{\Delta} \mathbf{u}_{n}\right),
\end{aligned}
$$

Moreover,

$$
c\left(t,-\widetilde{\Delta} \mathbf{u}_{n}\right)=\sum_{i=1}^{3} P_{i}(t) \int_{\Gamma_{i}} \mathbf{n} \cdot \widetilde{\Delta} \mathbf{u}_{n} d s .
$$

It follows that

$$
\begin{aligned}
\frac{1}{2} \frac{d}{d t}\left\|\nabla \mathbf{u}_{n}\right\|^{2}+\mu\left\|\widetilde{\Delta} \mathbf{u}_{n}\right\|^{2}= & \left(\left(\mathbf{u}_{n} \cdot \nabla\right) \mathbf{u}_{n}, \widetilde{\Delta} \mathbf{u}_{n}\right) \\
& +\sum_{i=1}^{3} P_{i}(t) \int_{\Gamma_{i}} \mathbf{n} \cdot\left(\widetilde{\Delta} \mathbf{u}_{n}\right) d s \\
& +\sum_{i=1}^{3} K_{i} \int_{\Gamma_{i}}\left(\mathbf{u}_{n} \cdot \mathbf{n}\right) \mathbf{n} \cdot \widetilde{\Delta} \mathbf{u}_{n} d s .
\end{aligned}
$$

We now make use of (28) to obtain

$$
\left|\sum_{i=1}^{3} P_{i}(t) \int_{\Gamma_{i}} \mathbf{n} \cdot\left(\widetilde{\Delta} \mathbf{u}_{n}\right) d s\right| \leq c_{3} \widetilde{P}\left\|\widetilde{\Delta} \mathbf{u}_{n}\right\|
$$


Likewise, from (29), we have

$$
\left|\sum_{i=1}^{3} K_{i} \int_{\Gamma_{i}}\left(\mathbf{u}_{n} \cdot \mathbf{n}\right) \mathbf{n} \cdot \widetilde{\Delta} \mathbf{u}_{n} d s\right| \leq c_{4} K\left\|\nabla \mathbf{u}_{n}\right\|\left\|\widetilde{\Delta} \mathbf{u}_{n}\right\| .
$$

Furthermore,

$$
\left(\left(\mathbf{u}_{n} \cdot \nabla\right) \mathbf{u}_{n}, \widetilde{\Delta} \mathbf{u}_{n}\right) \leq \sup _{\Omega}\left|\mathbf{u}_{n}\right|\left\|\nabla \mathbf{u}_{n}\right\|\left\|\widetilde{\Delta} \mathbf{u}_{n}\right\| .
$$

We make use of these inequalities in (78) to find that

$$
\begin{aligned}
\frac{1}{2} \frac{d}{d t}\left\|\nabla \mathbf{u}_{n}\right\|^{2}+\mu\left\|\widetilde{\Delta} \mathbf{u}_{n}\right\|^{2} \leq & \sup _{\Omega}\left|\mathbf{u}_{n}\right|\left\|\nabla \mathbf{u}_{n}\right\|\left\|\widetilde{\Delta} \mathbf{u}_{n}\right\| \\
& +c_{3} \widetilde{P}\left\|\widetilde{\Delta} \mathbf{u}_{n}\right\|+c_{4} K\left\|\nabla \mathbf{u}_{n}\right\|\left\|\widetilde{\Delta} \mathbf{u}_{n}\right\| .
\end{aligned}
$$

Now from (9),

$$
\sup _{\Omega}\left|\mathbf{u}_{n}\right| \leq c_{1}\left\|\nabla \mathbf{u}_{n}\right\|^{1 / 2}\left\|\widetilde{\Delta} \mathbf{u}_{n}\right\|^{1 / 2}
$$

which implies that

$$
\sup _{\Omega}\left|\mathbf{u}_{n}\right|\left\|\nabla \mathbf{u}_{n}\right\|\left\|\widetilde{\Delta} \mathbf{u}_{n}\right\| \leq c_{1}\left\|\nabla \mathbf{u}_{n}\right\|^{3 / 2}\left\|\widetilde{\Delta} \mathbf{u}_{n}\right\|^{3 / 2},
$$

and so, inequality (82) now reads

$$
\begin{aligned}
\frac{1}{2} \frac{d}{d t}\left\|\nabla \mathbf{u}_{n}\right\|^{2}+\mu\left\|\widetilde{\Delta} \mathbf{u}_{n}\right\|^{2} \leq & c_{1}\left\|\nabla \mathbf{u}_{n}\right\|^{3 / 2}\left\|\widetilde{\Delta} \mathbf{u}_{n}\right\|^{3 / 2} \\
& +c_{3} \widetilde{P}\left\|\widetilde{\Delta} \mathbf{u}_{n}\right\|+c_{4} K\left\|\nabla \mathbf{u}_{n}\right\|\left\|\widetilde{\Delta} \mathbf{u}_{n}\right\| .
\end{aligned}
$$

We make use of Cauchy's inequality with epsilon to estimate the right-hand side of (85). We have

$$
\begin{aligned}
& c_{1}\left\|\nabla \mathbf{u}_{n}\right\|^{3 / 2}\left\|\widetilde{\Delta} \mathbf{u}_{n}\right\|^{3 / 2}=\left(c_{1}\left\|\nabla \mathbf{u}_{n}\right\|^{3 / 2}\left\|\widetilde{\Delta} \mathbf{u}_{n}\right\|^{1 / 2}\right)\left\|\widetilde{\Delta} \mathbf{u}_{n}\right\| \\
& \leq \frac{1}{4 \epsilon_{1}} c_{1}^{2}\left\|\nabla \mathbf{u}_{n}\right\|^{3}\left\|\widetilde{\Delta} \mathbf{u}_{n}\right\|+\epsilon_{1}\left\|\widetilde{\Delta} \mathbf{u}_{n}\right\|^{2} \\
& \leq \frac{8 c_{1}^{4}}{\mu^{3}}\left\|\nabla \mathbf{u}_{n}\right\|^{6}+\frac{\mu}{4}\left\|\widetilde{\Delta} \mathbf{u}_{n}\right\|^{2}, \\
& c_{3} \widetilde{P}\left\|\widetilde{\Delta} \mathbf{u}_{n}\right\| \leq \frac{c_{3}^{2} \widetilde{P}^{2}}{\mu}+\frac{\mu}{4}\left\|\widetilde{\Delta} \mathbf{u}_{n}\right\|^{2} \quad\left(\operatorname{set} \epsilon=\frac{1}{\mu}\right) .
\end{aligned}
$$

Also inequality (10) implies that

$$
\left\|\nabla \mathbf{u}_{n}\right\| \leq c_{2}\left\|\widetilde{\Delta} \mathbf{u}_{n}\right\|
$$

so that

$$
c_{4} K\left\|\nabla \mathbf{u}_{n}\right\|\left\|\widetilde{\Delta} \mathbf{u}_{n}\right\| \leq K c_{2} c_{4}\left\|\widetilde{\Delta} \mathbf{u}_{n}\right\|^{2}
$$

Making use of theseinequalities on (85), we obtain that

$$
\begin{aligned}
\frac{1}{2} \frac{d}{d t}\left\|\nabla \mathbf{u}_{n}\right\|^{2}+\mu\left\|\widetilde{\Delta} \mathbf{u}_{n}\right\|^{2} \leq & \frac{8 c_{1}^{4}}{\mu^{3}}\left\|\nabla \mathbf{u}_{n}\right\|^{6}+\frac{\mu}{4}\left\|\widetilde{\Delta} \mathbf{u}_{n}\right\|^{2} \\
& +\frac{c_{3}^{2} \widetilde{P}^{2}}{\mu}+\frac{\mu}{4}\left\|\widetilde{\Delta} \mathbf{u}_{n}\right\|^{2}+K c_{2} c_{4}\left\|\widetilde{\Delta} \mathbf{u}_{n}\right\|^{2},
\end{aligned}
$$

which leads us to the required energy estimates.

4.4. Local Existence of a Weak Solution. In this subsection, we use Galerkin method to build up a local weak solution of the initial/boundary-value problem. We have already constructed the Galerkin approximations sequence $\left\{\mathbf{u}_{n}\right\}_{n=1}^{\infty}$ in Section 4.2. Our goal now is to extract from this sequence a subsequence that converges to the weak solution. In this respect, we are going to show that this sequence is bounded and thereafter, we will make use of a compactness result.

Lemma 9. Let $Y$ be a nonnegative function satisfying the inequality

$$
Y^{\prime} \leq \eta Y^{3}+\zeta
$$

and let $Y(0)=M_{0}$ be a strictly positive real number; then there exists a time interval $\left(0, T^{\star}\right)$ where $Y(t)$ is bounded by a positive constant $M$ and $M$ depends only on $M_{0}, \eta$ and $\zeta$.

Proof. We have

$$
Y^{\prime} \leq \eta(Y+\tau)^{3}, \quad \text { where } \tau=\left(\frac{\zeta}{\eta}\right)^{1 / 3} .
$$

We set $Z=Y+\tau$ to obtain

$$
\frac{Z^{\prime}}{Z^{3}} \leq \eta
$$

Integrate over $(0, t)$, with $0 \leq t \leq T$ :

$$
Z(t)^{-2} \geq Z(0)^{-2}-2 \eta T \text {. }
$$

We select $T^{\star}$ in $(0, T)$ such that $Z(0)^{-2}-2 \eta T^{\star}>0$; that is,

$$
T^{\star}<\frac{(Z(0))^{-2}}{2 \eta}=\frac{\left(M_{0}+(\zeta / \eta)^{1 / 3}\right)^{-2}}{2 \eta} .
$$

Then

$$
Z(t)^{-2}-Z(0)^{-2} \geq-2 \eta t \geq-2 \eta T^{\star}, \quad t \in\left(0, T^{\star}\right),
$$

so that

$$
Z(t) \leq\left(Z(0)^{-2}-2 \eta T^{\star}\right)^{-1 / 2}, \quad t \in\left(0, T^{\star}\right) .
$$

Considering that $Z=Y+\tau$ and $\tau=(\zeta / \eta)^{1 / 3}$, we obtain

$$
Y(t) \leq\left(\left(M_{0}+\left(\frac{\zeta}{\eta}\right)^{1 / 3}\right)^{-2}-2 \eta T^{\star}\right)^{-1 / 2}
$$

$$
-\left(\frac{\zeta}{\eta}\right)^{1 / 3}, \quad t \in\left(0, T^{\star}\right) .
$$


In order to obtain $\left(\left(M_{0}+(\zeta / \eta)^{1 / 3}\right)^{-2}-2 \eta T^{\star}\right)^{-1 / 2}-(\zeta / \eta)^{1 / 3}>$ 0 , we must have

$$
T^{\star}>\frac{\left(M_{0}+(\zeta / \eta)^{1 / 3}\right)^{-2}-(\zeta / \eta)^{-2 / 3}}{2 \eta} .
$$

Hence, we choose $T^{\star}$ such that

$$
\frac{\left(M_{0}+(\zeta / \eta)^{1 / 3}\right)^{-2}-(\zeta / \eta)^{-2 / 3}}{2 \eta}<T^{\star}<\frac{\left(M_{0}+(\zeta / \eta)^{1 / 3}\right)^{-2}}{2 \eta} .
$$

It follows that $Y(t)$ is bounded by a positive constant

$$
M=\left(\left(M_{0}+\left(\frac{\zeta}{\eta}\right)^{1 / 3}\right)^{-2}-2 \eta T^{\star}\right)^{-1 / 2}-\left(\frac{\zeta}{\eta}\right)^{1 / 3}
$$

for all $t$ such that $0 \leq t \leq T^{\star}$ and that $M$ depends only on $M_{0}$, $\eta$, and $\zeta$.

Theorem 10. Let $K$ be such that

$$
\kappa=\mu-2 K c_{2} c_{4}>0 ;
$$

there is a time interval $\left(0, T^{\star}\right)$ on which a weak solution of (23) exists.

Proof. Considering (101), the energy estimates (73) now read:

$$
\frac{d}{d t}\left\|\nabla \mathbf{u}_{n}\right\|^{2}+\kappa\left\|\widetilde{\Delta} \mathbf{u}_{n}\right\|^{2} \leq \frac{16 c_{1}^{4}}{\mu^{3}}\left\|\nabla \mathbf{u}_{n}\right\|^{6}+\frac{2 c_{3}^{2} \widetilde{P}^{2}}{\mu} .
$$

Since $\kappa>0$, (102) implies that

$$
\frac{d}{d t}\left\|\nabla \mathbf{u}_{n}\right\|^{2} \leq \frac{16 c_{1}^{4}}{\mu^{3}}\left\|\nabla \mathbf{u}_{n}\right\|^{6}+\frac{2 c_{3}^{2} \widetilde{P}^{2}}{\mu} .
$$

Defining

$$
Y_{n}(t)=\left\|\nabla \mathbf{u}_{n}\right\|^{2}
$$

we see that inequality (103) has the following form:

$$
Y_{n}^{\prime} \leq \eta Y_{n}^{3}+\zeta
$$

where $\eta>0$ and $\zeta>0$ are some constants depending on the data.

Note that for each integer $n$, the function $Y_{n}(t)=\left\|\nabla \mathbf{u}_{n}\right\|^{2}$ verifies the requirements of Lemma 9 with $Y_{n}(0)=\left\|\nabla \mathbf{u}_{n}\right\|^{2}$ since the initial condition is the same for all functions $\mathbf{u}_{n}$, $n=0,1,2, \ldots$. Consequently, the sequence $\left\{\left\|\nabla \mathbf{u}_{n}\right\|^{2}\right\}_{n=1}^{\infty}$ is bounded by a real number $M$ which depends only on the initial data. We make use of Poincarés inequality (4) to find that for each integer $n$,

$$
\begin{gathered}
\left\|\mathbf{u}_{n}\right\|_{1}^{2}=\left\|\mathbf{u}_{n}\right\|^{2}+\left\|\nabla \mathbf{u}_{n}\right\|^{2} \leq\left(1+C_{\Omega}^{2}\right) M, \\
\left\|\mathbf{u}_{n}\right\|_{L^{2}\left(0, T^{\star} ; \mathbf{V}\right)}^{2}=\int_{0}^{T^{\star}}\left\|\mathbf{u}_{n}\right\|_{1}^{2} d t \leq\left(1+C_{\Omega}^{2}\right) M T^{\star} .
\end{gathered}
$$

Therefore, the sequence $\left\{\left\|\mathbf{u}_{n}\right\|_{L^{2}\left(0, T^{\star} ; \mathbf{V}\right)}\right\}_{n=1}^{\infty}$ is bounded.

Furthermore, the space $L^{2}\left(0, T^{\star} ; \mathbf{V}\right)$ being a Hilbert space, Theorem 4 can be applied to the sequence $\left\{\left\|\mathbf{u}_{n}\right\|_{L^{2}\left(0, T^{\star} ; \mathbf{V}\right)}\right\}_{n=1}^{\infty}$.
In fact, the sequence $\left\{\mathbf{u}_{n}\right\}_{n=1}^{\infty}$ is bounded in $L^{2}\left(0, T^{\star} ; \mathbf{V}\right)$. Consequently, there exists a subsequence $\left\{\mathbf{u}_{n_{q}}\right\}_{q=1}^{\infty} \subset\left\{\mathbf{u}_{n}\right\}_{n=1}^{\infty}$ such that

$$
\mathbf{u}_{n_{q}} \rightarrow \mathbf{u} \quad \text { weakly in } \mathbf{L}^{2}\left(0, T^{\star} ; \mathbf{V}\right)
$$

Next, we show that the weak limit $\mathbf{u}$ is in fact a weak solution. In this respect, we are going to show first that

$$
\left(\frac{\partial \mathbf{u}}{\partial t}, \boldsymbol{\varphi}\right)+b(\mathbf{u}, \mathbf{u}, \boldsymbol{\varphi})+a(\mathbf{u}, \boldsymbol{\varphi})=c(t, \boldsymbol{\varphi}), \quad \forall \boldsymbol{\varphi} \in \mathbf{V}
$$

and, thereafter,

$$
(\mathbf{u}(0), \varphi)=\left(\mathbf{u}_{0}, \varphi\right), \quad \forall \varphi \in \mathbf{V} .
$$

(1) Fix an integer $N$ and choose a function $\mathbf{w} \in \mathbf{C}\left(0, T^{\star}\right.$; V) having the form

$$
\mathbf{w}(t)=\sum_{k=1}^{N} d^{k}(t) \mathbf{a}_{k}
$$

where $\left\{d^{k}\right\}_{k=1}^{N}$ are given functions and $\left\{\mathbf{a}_{k}\right\}$ is the basis of $\mathbf{V}$. We choose $n \geq N$, multiply (67) by $d^{k}(t)$, sum $k=1, \ldots, N$, and then integrate with respect to $t$ to find that

$$
\begin{aligned}
& \int_{0}^{T^{\star}} {\left[\left(\frac{\partial \mathbf{u}_{n_{q}}}{\partial t}, \mathbf{w}\right)+a\left(\mathbf{u}_{n_{q}}, \mathbf{w}\right)+b\left(\mathbf{u}_{n_{q}}, \mathbf{u}_{n_{q}}, \mathbf{w}\right)\right] d t } \\
& \quad=\int_{0}^{T^{\star}} c(t, \mathbf{w}) d t .
\end{aligned}
$$

We recall (107) to find upon passing to weak limits (see remark 3.1 and [5]) that

$$
\begin{aligned}
\int_{0}^{T^{\star}} & {\left[\left(\frac{\partial \mathbf{u}}{\partial t}, \mathbf{w}\right)+a(\mathbf{u}, \mathbf{w})+b(\mathbf{u}, \mathbf{u}, \mathbf{w})\right] d t } \\
& =\int_{0}^{T^{\star}} c(t, \mathbf{w}) d t .
\end{aligned}
$$

Equality (112) then holds for all functions $\mathbf{w} \in \mathbf{L}^{2}(0$, $T ; \mathbf{V})$, as functions of the form (110) are dense in this space [5]. Hence, in particular,

$$
\left(\frac{\partial \mathbf{u}}{\partial t}, \boldsymbol{\varphi}\right)+b(\mathbf{u}, \mathbf{u}, \boldsymbol{\varphi})+a(\mathbf{u}, \boldsymbol{\varphi})=c(t, \boldsymbol{\varphi})
$$

for each $\varphi \in \mathbf{V}$ and a.e. $t \geq 0$.

(2) In order to prove that $(\mathbf{u}(0), \varphi)=\left(\mathbf{u}_{0}, \boldsymbol{\varphi}\right)$ for every $\varphi \in \mathrm{V}$, we first note from (112) that

$$
\begin{gathered}
\int_{0}^{T^{\star}}\left[-\left(\mathbf{u}, \frac{\partial \mathbf{w}}{\partial t}\right)+a(\mathbf{u}, \mathbf{w})+b(\mathbf{u}, \mathbf{u}, \mathbf{w})\right] d t \\
\quad=\int_{0}^{T^{\star}} c(t, \mathbf{w}) d t+(\mathbf{u}(0), \mathbf{w}(0)),
\end{gathered}
$$


for each $\mathbf{w} \in \mathbf{H}^{1}\left(0, T^{\star} ; \mathbf{V}\right)$ with $\mathbf{w}\left(T^{\star}\right)=0$. Similarly, from (111) we deduce that

$$
\begin{aligned}
& \int_{0}^{T^{\star}}\left[-\left(\mathbf{u}_{n_{q}}, \frac{\partial \mathbf{w}}{\partial t}\right)+a\left(\mathbf{u}_{n_{q}}, \mathbf{w}\right)+b\left(\mathbf{u}_{n_{q}}, \mathbf{u}_{n_{q}}, \mathbf{w}\right)\right] d t \\
& \quad=\int_{0}^{T^{\star}} c(t, \mathbf{w}) d t+\left(\mathbf{u}_{n_{q}}(0), \mathbf{w}(0)\right) .
\end{aligned}
$$

We once again employ (107) to find that

$$
\begin{aligned}
& \int_{0}^{T^{\star}}\left[-\left(\mathbf{u}, \frac{\partial \mathbf{w}}{\partial t}\right)+a(\mathbf{u}, \mathbf{w})+b(\mathbf{u}, \mathbf{u}, \mathbf{w})\right] d t \\
& \quad=\int_{0}^{T^{\star}} c(t, \mathbf{w}) d t+\left(\mathbf{u}_{0}, \mathbf{w}(0)\right),
\end{aligned}
$$

since $\mathbf{u}_{n_{q}}(0) \rightarrow \mathbf{u}_{0}$. As $\mathbf{w}(0)$ is arbitrary, comparing (114) and (116), we conclude that $(\mathbf{u}(0), \varphi)=\left(\mathbf{u}_{0}, \varphi\right)$ for each $\varphi \in \mathbf{V}$. Therefore, $\mathbf{u}$ is a local weak solution of (23).

4.5. Global Existence of a Weak Solution for Small Data. In this section, we make use of Galerkin's method to establish the global existence of a weak solution. We are going to show that under certain circumstances, the weak solution $\mathbf{u}$ is defined at any time $t$. In this regard, we are going to consider the energy estimate and the following lemma.

Lemma 11. Let $Y$ be a nonnegative, absolutely continuous function satisfying inequality

$$
Y^{\prime}+\lambda Y \leq \eta Y^{3}+\zeta
$$

and let a real number $M$ be such that $0<M<(\lambda / 2 \eta)^{1 / 2}$, if $Y(0) \leq M$, and $\zeta \leq \lambda M / 2$, then $Y(t)$ is bounded by $M$ for all $t>0$.

Proof. This result is proven by contradiction [8]. Suppose that there exists a $t$ such that $Y(t)>M$, and define

$$
t^{\star}=\inf \left\{t \in \mathbb{R}_{+}, Y(t)>M\right\} ;
$$

we have $Y\left(t^{\star}\right)=M$ and $Y^{\prime}\left(t^{\star}\right)>0$.

(1) We first show that $Y\left(t^{\star}\right)=M$. Set $I=\left\{t \in \mathbb{R}_{+}, Y(t)>\right.$ $M\}$ and choose any $\epsilon>0$; we have

$$
t^{\star}-\epsilon<t^{\star}, \quad t^{\star}=\inf (I),
$$

and so $t^{\star}-\epsilon$ does not belong to the set $I$. It follows that $Y\left(t^{\star}-\epsilon\right) \leq M$, and this is true for each $\epsilon>0$; hence

$$
Y\left(t^{\star}\right) \leq M
$$

On the other hand, for each natural number $n$, we have $t^{\star}+(1 / n)>t^{\star}$. We make use of the fact that $t^{\star}=\inf (I)$ to see that there exists $\phi_{n} \in I$ such that

$$
t^{\star} \leq \phi_{n} \leq t^{\star}+\frac{1}{n}
$$

where $\phi_{n}$ is not necessarily unique. We choose one value that $\phi_{n}$ may take and we denote it by $t_{n}$. This defines a real sequence $\left\{t_{n}\right\}_{n=1}^{\infty}$. For each natural number $n$, since $t_{n} \in I$, we have that $Y\left(t_{n}\right)>M$. The fact that $t^{\star} \leq t_{n} \leq t^{\star}+(1 / n)$ implies that the sequence $\left\{t_{n}\right\}_{n=1}^{\infty}$ converges to $t^{\star}$. We make use of the continuity of $Y$ to see that the sequence

$$
\left\{Y\left(t_{n}\right)\right\}_{n=1}^{\infty} \text { converges to } Y\left(t^{\star}\right) .
$$

Since $t_{n} \in I$ for each $n$, we have $Y\left(t_{n}\right)>M$, and then $Y\left(t_{n}\right) \rightarrow Y\left(t^{\star}\right)$ implies that

$$
Y\left(t^{\star}\right) \geq M
$$

It follows that $Y\left(t^{\star}\right) \leq M$ and $Y\left(t^{\star}\right) \geq M$; therefore,

$$
Y\left(t^{\star}\right)=M
$$

(2) Next we show that $Y^{\prime}\left(t^{\star}\right)>0$. Suppose that $Y^{\prime}\left(t^{\star}\right) \leq$ 0 ; then there exists a nonnegative natural number $n$ such that $Y$ decreases on the interval $\left(t^{\star}, t^{\star}+(1 / n)\right)$. Also we have that

$$
t^{\star} \leq t_{n} \leq t^{\star}+\frac{1}{n}
$$

this implies that

$$
Y\left(t^{\star}\right) \geq Y\left(t_{n}\right)
$$

But from part (1), we have $Y\left(t^{\star}\right)=M$ and $Y\left(t_{n}\right)>M$; because $t_{n} \in I$, it follows that

$$
Y\left(t^{\star}\right)<Y\left(t_{n}\right) \text {. }
$$

Hence, $Y\left(t^{\star}\right) \geq Y\left(t_{n}\right)$ and $Y\left(t^{\star}\right)<Y\left(t_{n}\right)$, which is impossible. Therefore,

$$
Y^{\prime}\left(t^{\star}\right)>0 \text {. }
$$

Finally that we show that $Y^{\prime}\left(t^{\star}\right) \leq 0$ and this contradicts the result obtained in (2). From (117), we have

$$
\begin{aligned}
Y^{\prime}\left(t^{\star}\right) & \leq-\lambda Y\left(t^{\star}\right)+\eta Y^{3}\left(t^{\star}\right)+\zeta \\
& \left.=-\lambda M+\eta M\left(M^{2}\right)+\zeta \quad \text { (because } Y\left(t^{\star}\right)=M\right) \\
& \leq-\lambda M+\eta M\left(\frac{\lambda}{2 \eta}\right)+\zeta \\
& \leq 0, \quad \text { because of the hypothesis }\left(\zeta \leq \frac{\lambda M}{2}\right) .
\end{aligned}
$$

Therefore, $Y(t) \leq M$ for all $t \in \mathbb{R}_{+}$.

Theorem 12. Assume that the initial and boundary data are sufficiently small, precisely

$$
\left\|\nabla \mathbf{u}_{0}\right\| \leq\left(\frac{\kappa \mu^{3}}{64 c_{2} c_{1}^{4}}\right)^{1 / 4}, \quad \widetilde{P}<\frac{\mu}{4 c_{1} c_{3}}\left(\frac{\kappa^{3} \mu}{4 c_{2}^{3}}\right)^{1 / 4} .
$$


Then, for all $T \geq 0$, there exists a weak solution $\mathbf{u} \in \mathbf{L}^{2}(0, T ; \mathbf{V})$ to problem (23), and it satisfies the inequality

$$
\|\nabla \mathbf{u}\| \leq\left(\frac{\kappa \mu^{3}}{64 c_{2} c_{1}^{4}}\right)^{1 / 4}
$$

Proof. We make use of the estimates (10), (73), and (101) in order to obtain

$$
\frac{d}{d t}\left\|\nabla \mathbf{u}_{n}\right\|^{2}+\frac{\kappa}{c_{2}}\left\|\nabla \mathbf{u}_{n}\right\|^{2} \leq \frac{16 c_{1}^{4}}{\mu^{3}}\left\|\nabla \mathbf{u}_{n}\right\|^{6}+\frac{2 c_{3}^{2} \widetilde{P}^{2}}{\mu} .
$$

Set

$$
Y_{n}=\left\|\nabla \mathbf{u}_{n}\right\|^{2}
$$

The energy estimate (132) takes the form

$$
Y_{n}^{\prime}+\lambda Y_{n} \leq \eta Y_{n}^{3}+\zeta
$$

where

$$
\lambda=\frac{\kappa}{c_{2}}, \quad \eta=\frac{16 c_{1}^{4}}{\mu^{3}}, \quad \zeta=\frac{2 c_{3}^{2} \widetilde{P}^{2}}{\mu} .
$$

Let

$$
M=\left(\frac{\kappa \mu^{3}}{64 c_{2} c_{1}^{4}}\right)^{1 / 2}
$$

we have

$$
0<M<\left(\frac{\lambda}{2 \eta}\right)^{1 / 2}
$$

Also

$$
\begin{gathered}
Y_{n}(0)=\left\|\nabla \mathbf{u}_{0}\right\|^{2} \leq M \quad \text { for each integer } n, \\
\zeta=\frac{2 c_{3}^{2} \widetilde{P}^{2}}{\mu} \leq \frac{\kappa}{2 c_{2}}\left(\frac{\kappa \mu^{3}}{64 c_{2} c_{1}^{4}}\right)^{1 / 2}=\frac{\lambda M}{2} .
\end{gathered}
$$

We make use of Lemma 11 to find that $Y_{n}=\left\|\nabla \mathbf{u}_{n}\right\|^{2}$ is bounded by $M$ for all $t>0$ and the bound does not depend on $n$. Poincaré's inequality (4) implies that

$$
\begin{aligned}
\left\|\mathbf{u}_{n}\right\|_{1}^{2} & =\left\|\mathbf{u}_{n}\right\|^{2}+\left\|\nabla \mathbf{u}_{n}\right\|^{2} \\
& \leq C_{\Omega}^{2}\left\|\nabla \mathbf{u}_{n}\right\|^{2}+\left\|\nabla \mathbf{u}_{n}\right\|^{2} \leq\left(1+C_{\Omega}^{2}\right) M .
\end{aligned}
$$

Let $T>0$ be any positive real number,

$$
\left\|\mathbf{u}_{n}\right\|_{L^{2}(0, T ; \mathbf{V})}^{2}=\int_{0}^{T}\left\|\mathbf{u}_{n}\right\|_{1}^{2} d t \leq\left(1+C_{\Omega}^{2}\right) M T .
$$

Thus, for each nonnegative real number $T$, the sequence $\left\{\left\|\mathbf{u}_{n}\right\|_{L^{2}(0, T ; \mathbf{V})}\right\}_{n=1}^{\infty}$ is bounded. We make use of Lemma 9 to extract a subsequence $\left\{\mathbf{u}_{n q}\right\}_{q=1}^{\infty}$ that converges to an element $\mathbf{u}$ of $L^{2}(0, T ; \mathbf{V})$, and we use the same steps as we did for the local existence to show that $\mathbf{u}$ is a weak solution of problem (23).
Furthermore, the fact that the sequence $\left\{\nabla \mathbf{u}_{n}\right\}_{n=1}^{\infty}$ is bounded by $\sqrt{M}$ implies that the subsequence $\left\{\nabla \mathbf{u}_{n q}\right\}_{q=1}^{\infty}$ is also bounded by $\sqrt{M}$.

Therefore, since $\nabla \mathbf{u}$ is the limit of this subsequence, it follows that

$$
\|\nabla \mathbf{u}\| \leq\left(\frac{\kappa \mu^{3}}{64 c_{2} c_{1}^{4}}\right)^{1 / 4}
$$

\section{Uniqueness of the Solution}

Theorem 13. There exists a time interval $T_{1}$ where the solution of problem (23) is unique.

Proof. Assume that there exist two solutions $\mathbf{u}^{(1)}$ and $\mathbf{u}^{(2)}$ associated with the same data. Set $\mathbf{w}=\mathbf{u}^{(1)}-\mathbf{u}^{(2)}$. Consider (63) with $\varphi=\mathbf{w}$. By substraction, we obtain

$$
\left(\frac{\partial \mathbf{w}}{\partial t}, \mathbf{w}\right)+a(\mathbf{w}, \mathbf{w})=b\left(\mathbf{u}^{(2)}, \mathbf{u}^{(2)}, \mathbf{w}\right)-b\left(\mathbf{u}^{(1)}, \mathbf{u}^{(1)}, \mathbf{w}\right)
$$

We recall that $a$ is a bilinear form, $b$ is a trilinear form, and $c$ is a linear form described by (24), (25), and (26), respectively. We evaluate every term of (142):

$$
\left(\frac{\partial \mathbf{w}}{\partial t}, \mathbf{w}\right)=\frac{1}{2} \frac{d}{d t}\|\mathbf{w}\|^{2}
$$

$$
a(\mathbf{w}, \mathbf{w})=\mu\|\nabla \mathbf{w}\|^{2}+\sum_{i=1}^{3} K_{i} \int_{\Gamma i}|\mathbf{w} \cdot \mathbf{n}|^{2} d s,
$$

and also,

$$
\begin{aligned}
& b\left(\mathbf{u}^{(2)}, \mathbf{u}^{(2)}, \mathbf{w}\right)-b\left(\mathbf{u}^{(1)}, \mathbf{u}^{(1)}, \mathbf{w}\right) \\
&= b\left(\mathbf{u}^{(2)}, \mathbf{u}^{(2)}, \mathbf{w}\right) \\
&-b\left(\mathbf{u}^{(2)}, \mathbf{u}^{(2)}+\mathbf{w}, \mathbf{w}\right)-b\left(\mathbf{w}, \mathbf{u}^{(2)}+\mathbf{w}, \mathbf{w}\right) \\
&=-b(\mathbf{w}, \mathbf{w}, \mathbf{w})-b\left(\mathbf{u}^{(2)}, \mathbf{w}, \mathbf{w}\right)-b\left(\mathbf{w}, \mathbf{u}^{(2)}, \mathbf{w}\right) .
\end{aligned}
$$

It follows that

$$
\begin{aligned}
& \frac{1}{2} \frac{d}{d t}\|\mathbf{w}\|^{2}+\mu\|\nabla \mathbf{w}\|^{2}+\sum_{i=1}^{3} K_{i} \int_{\Gamma i}|\mathbf{w} \cdot \mathbf{n}|^{2} d s \\
& \quad=-b(\mathbf{w}, \mathbf{w}, \mathbf{w})-b\left(\mathbf{u}^{(2)}, \mathbf{w}, \mathbf{w}\right)-b\left(\mathbf{w}, \mathbf{u}^{(2)}, \mathbf{w}\right) .
\end{aligned}
$$

We now make use of some classical estimates to evaluate the rights hand side of (145) as follow: 


$$
\begin{aligned}
b(\mathbf{w}, \mathbf{w}, \mathbf{w})= & ((\mathbf{w} \cdot \nabla) \mathbf{w}, \mathbf{w}) \\
= & -\int_{\Omega} \sum_{i=1}^{3}\left(\sum_{j=1}^{3} \frac{\partial\left(w_{i} w_{j}\right)}{\partial x_{j}}\right) w_{i} d \mathbf{x} \\
& +\int_{\Gamma}(\mathbf{w} \cdot \mathbf{w})(\mathbf{w} \cdot \mathbf{n}) d s=-b(\mathbf{w}, \mathbf{w}, \mathbf{w}) \\
& +\int_{\Gamma}(\mathbf{w} \cdot \mathbf{w})(\mathbf{w} \cdot \mathbf{n}) d s, \quad \text { because } \nabla \cdot \mathbf{w}=0 .
\end{aligned}
$$

This implies that

$$
\begin{aligned}
b(\mathbf{w}, \mathbf{w}, \mathbf{w}) & =\frac{1}{2} \int_{\Gamma}(\mathbf{w} \cdot \mathbf{w})(\mathbf{w} \cdot \mathbf{n}) d s \\
& \leq \frac{1}{2}\left(\int_{\Gamma}(\mathbf{w} \cdot \mathbf{w})^{2} d s\right)^{1 / 2}\left(\int_{\Gamma}(\mathbf{w} \cdot \mathbf{n})^{2} d s\right)^{1 / 2} \\
& \leq c_{5}\|\mathbf{w}\|_{L^{4}}^{2}\|\mathbf{w}\| \\
& \leq c_{6}\|\mathbf{w}\|\|\nabla \mathbf{w}\|^{2}
\end{aligned}
$$

Also,

$$
\begin{aligned}
&\left|b\left(\mathbf{u}^{(2)}, \mathbf{w}, \mathbf{w}\right)\right| \leq \sup _{\Omega}\left|\mathbf{u}^{(2)}\right|\|\nabla \mathbf{w}\|\|\mathbf{w}\| \\
& \leq \frac{\mu}{2}\|\nabla \mathbf{w}\|^{2}+\frac{1}{2 \mu}\left(\sup _{\Omega}\left|\mathbf{u}^{(2)}\right|\right)^{2}\|\mathbf{w}\|^{2}, \\
&\left|b\left(\mathbf{w}, \mathbf{u}^{(2)}, \mathbf{w}\right)\right| \leq \sup _{\Omega}\left|\nabla \mathbf{u}^{(2)}\right|\|\mathbf{w}\|^{2} .
\end{aligned}
$$

It follows that

$$
\begin{aligned}
\left|b\left(\mathbf{u}^{(2)}, \mathbf{w}, \mathbf{w}\right)+b\left(\mathbf{w}, \mathbf{u}^{(2)}, \mathbf{w}\right)\right| \\
\leq \frac{\mu}{2}\|\nabla \mathbf{w}\|^{2}+\frac{1}{2 \mu}\left(\sup _{\Omega}\left|\mathbf{u}^{(2)}\right|\right)^{2}\|\mathbf{w}\|^{2} \\
\quad+\sup _{\Omega}\left|\nabla \mathbf{u}^{(2)}\right|\|\mathbf{w}\|^{2} .
\end{aligned}
$$

We now make use of these estimates in (145) to find that

$$
\begin{gathered}
\frac{1}{2} \frac{d}{d t}\|\mathbf{w}\|^{2}+\mu\|\nabla \mathbf{w}\|^{2}+\sum_{i=1}^{3} K_{i} \int_{\Gamma i}|\mathbf{w} \cdot \mathbf{n}|^{2} d s \\
\leq \frac{\mu}{2}\|\nabla \mathbf{w}\|^{2}+\frac{1}{2 \mu}\left(\sup _{\Omega}\left|\mathbf{u}^{(2)}\right|\right)^{2}\|\mathbf{w}\|^{2} \\
+\sup _{\Omega}\left|\nabla \mathbf{u}^{(2)}\right|\|\mathbf{w}\|^{2}+c_{6}\|\mathbf{w}\|\|\nabla \mathbf{w}\|^{2} .
\end{gathered}
$$

Thus,

$$
\begin{aligned}
& \frac{d}{d t}\|\mathbf{w}\|^{2}+\left(\mu-2 c_{6}\|\mathbf{w}\|\right)\|\nabla \mathbf{w}\|^{2} \\
& \quad \leq\left(\frac{1}{\mu}\left(\sup _{\Omega}\left|\mathbf{u}^{(2)}\right|\right)^{2}+2\left(\sup _{\Omega}\left|\nabla \mathbf{u}^{(2)}\right|\right)\right)\|\mathbf{w}\|^{2} .
\end{aligned}
$$

Since $\mathbf{w}(0, \mathbf{x})=0$, there exists $T_{1}>0$ such that $\|\mathbf{w}\| \leq \mu / 2 c_{6}$ with $0<T_{1}<T^{\star}$. On the interval $\left(0, T_{1}\right)$, we have

$$
\frac{d}{d t}\|\mathbf{w}\|^{2} \leq \vartheta(t)\|\mathbf{w}\|^{2}
$$

where

$$
\vartheta(t)=\left(\frac{1}{\mu}\left(\sup _{\Omega}\left|\mathbf{u}^{(2)}\right|\right)^{2}+2\left(\sup _{\Omega}\left|\nabla \mathbf{u}^{(2)}\right|\right)\right)
$$

is a real function depending on $t$.

The uniqueness theorem follows from Gronwall's inequality [6], as $\mathbf{w}(0, \mathbf{x})=0$.

\section{Stability of the Solution}

In this section, we are going to prove that there exists a time interval of continuous dependence on the data. We start with the boundary conditions and afterward we move on to the initial condition.

\subsection{Boundary Conditions Stability}

Theorem 14. There exists a time interval where the solution of problem (23) depends continuously on the prescribed data as the real functions $P_{i}(i=1,2,3)$ are varied.

Proof. Denote by $\mathbf{u}^{(1)}$ the solution associated with the data $P_{i}^{(1)}(i=1,2,3)$ and, correspondingly, by $\mathbf{u}^{(2)}$ the solution associated with $P_{i}^{(2)}(i=1,2,3)$.

Set

$$
\mathbf{w}=\mathbf{u}^{(1)}-\mathbf{u}^{(2)}, \quad \bar{P}=\max _{i=1,2,3}\left|P_{i}^{(1)}-P_{i}^{(2)}\right| .
$$

We make use of (63) for $\mathbf{u}^{(1)}$ and $\mathbf{u}^{(2)}$ with $\boldsymbol{\varphi}=\mathbf{w}$. By substraction, we obtain

$$
\begin{aligned}
\left(\frac{\partial \mathbf{w}}{\partial t}, \mathbf{w}\right)+a(\mathbf{w}, \mathbf{w})= & b\left(\mathbf{u}^{(2)}, \mathbf{u}^{(2)}, \mathbf{w}\right)-b\left(\mathbf{u}^{(1)}, \mathbf{u}^{(1)}, \mathbf{w}\right) \\
& +c_{1}(t, \mathbf{w})-c_{2}(t, \mathbf{w}),
\end{aligned}
$$

where

$$
\begin{aligned}
& c_{j}(t, \mathbf{v})=-\sum_{i=1}^{3} P_{i}^{(j)}(t) \int_{\Gamma_{i}} \mathbf{n} \cdot \mathbf{v} d s \\
& \text { for each } \mathbf{v} \in \mathbf{H}^{1}(\Omega), \quad j=1,2 .
\end{aligned}
$$

This gives us that

$$
\begin{aligned}
\frac{1}{2} \frac{d}{d t}\|\mathbf{w}\|^{2}+\mu\|\nabla \mathbf{w}\|^{2}+\sum_{i=1}^{3} K_{i} \int_{\Gamma i}|\mathbf{w} \cdot \mathbf{n}|^{2} d s \\
=-b(\mathbf{w}, \mathbf{w}, \mathbf{w})-b\left(\mathbf{u}^{(2)}, \mathbf{w}, \mathbf{w}\right)-b\left(\mathbf{w}, \mathbf{u}^{(2)}, \mathbf{w}\right) \\
\quad+\sum_{i=1}^{3}\left(P_{i}^{(2)}-P_{i}^{(1)}\right) \int_{\Gamma i} \mathbf{w} \cdot \mathbf{n} d s .
\end{aligned}
$$


Also,

$$
\begin{aligned}
& \sum_{i=1}^{3}\left(P_{i}^{(2)}-P_{i}^{(1)}\right) \int_{\Gamma i} \mathbf{w} \cdot \mathbf{n} d s \leq \sum_{i=1}^{3} \bar{P} \int_{\Gamma i}|\mathbf{w} \cdot \mathbf{n}| d s \\
& \leq c_{7} \bar{P}^{2}+\sum_{i=1}^{3} K_{i} \int_{\Gamma i}|\mathbf{w} \cdot \mathbf{n}|^{2} d s .
\end{aligned}
$$

It follows that

$$
\begin{aligned}
& \frac{d}{d t}\|\mathbf{w}\|^{2}+\left(\mu-2 c_{6}\|\mathbf{w}\|\right)\|\nabla \mathbf{w}\|^{2} \\
& \quad \leq\left(\frac{1}{\mu}\left(\sup _{\Omega}\left|\mathbf{u}^{(2)}\right|\right)^{2}+2\left(\sup _{\Omega}\left|\nabla \mathbf{u}^{(2)}\right|\right)\right)\|\mathbf{w}\|^{2}+2 c_{7} \bar{P}^{2} .
\end{aligned}
$$

Since $\mathbf{w}(0, \mathbf{x})=0$, there exists $T_{1}>0$ such that $\|\mathbf{w}\| \leq \mu / 2 c_{6}$ with $0<T_{1}<T^{\star}$. On the interval $\left(0, T_{1}\right)$, we have that

$$
\frac{d}{d t}\|\mathbf{w}\|^{2} \leq c_{8}\|\mathbf{w}\|^{2}+2 c_{7} \bar{P}^{2},
$$

where

$$
c_{8}=\sup _{\left(0, T_{1}\right)}\left[\left(\frac{1}{\mu}\left(\sup _{\Omega}\left|\mathbf{u}^{(2)}\right|\right)^{2}+2\left(\sup _{\Omega}\left|\nabla \mathbf{u}^{(2)}\right|\right)\right)\right] .
$$

From Gronwall's inequality [6], we deduce that in the interval $\left(0, T_{1}\right)$

$$
\|\mathbf{w}\| \leq c \max _{0<t \leq T_{1}} \bar{P}, \quad \text { where } \bar{P}=\max _{i=1,2,3}\left|P_{i}^{(1)}-P_{i}^{(2)}\right| .
$$

Hence, a small change in the given data produces a correspondingly small change in the solution.

Therefore, the solution of problem (23) is stable in the interval $\left(0, T_{1}\right)$ as the real functions $P_{i}(i=1,2,3)$ are varied.

\subsection{Initial Condition Stability}

Theorem 15. There exists a time interval where the solution of problem (23) depends continuously on the prescribed data as the initial condition $\mathbf{u}_{0}(\mathbf{x})$ is varied.

Proof. Denote by $\mathbf{u}^{(1)}$ the solution associated with the initial data $\mathbf{u}_{0}^{(1)}$ and, correspondingly, by $\mathbf{u}^{(2)}$ the solution associated with the initial data $\mathbf{u}_{0}^{(2)}$.

Set $\mathbf{w}=\mathbf{u}^{(1)}-\mathbf{u}^{(2)}$.

Consider (63) with $\varphi=\mathbf{w}$. By substraction, we obtain

$$
\left(\frac{\partial \mathbf{w}}{\partial t}, \mathbf{w}\right)+a(\mathbf{w}, \mathbf{w})=b\left(\mathbf{u}^{(2)}, \mathbf{u}^{(2)}, \mathbf{w}\right)-b\left(\mathbf{u}^{(1)}, \mathbf{u}^{(1)}, \mathbf{w}\right),
$$

and this gives us

$$
\begin{aligned}
\frac{1}{2} & \frac{d}{d t}\|\mathbf{w}\|^{2}+\mu\|\nabla \mathbf{w}\|^{2}+\sum_{i=1}^{3} K_{i} \int_{\Gamma i}|\mathbf{w} \cdot \mathbf{n}|^{2} d s \\
& =-b(\mathbf{w}, \mathbf{w}, \mathbf{w})-b\left(\mathbf{u}^{(2)}, \mathbf{w}, \mathbf{w}\right)-b\left(\mathbf{w}, \mathbf{u}^{(2)}, \mathbf{w}\right) .
\end{aligned}
$$

We estimate the right-hand side of this equality like we did in the case of uniqueness and we obtain

$$
\begin{aligned}
\frac{1}{2} \frac{d}{d t}\|\mathbf{w}\|^{2}+\mu\|\nabla \mathbf{w}\|^{2}+\sum_{i=1}^{3} K_{i} \int_{\Gamma i}|\mathbf{w} \cdot \mathbf{n}|^{2} d s \\
\leq \frac{\mu}{2}\|\nabla \mathbf{w}\|^{2}+\frac{1}{2 \mu}\left(\left(\sup _{\Omega}\left|\mathbf{u}^{(2)}\right|\right)^{2}\|\mathbf{w}\|^{2}\right. \\
+\sup _{\Omega}\left|\nabla \mathbf{u}^{(2)}\right|\|\mathbf{w}\|^{2}+c_{6}\|\mathbf{w}\|\|\nabla \mathbf{w}\|^{2} .
\end{aligned}
$$

Thus,

$$
\begin{aligned}
& \frac{d}{d t}\|\mathbf{w}\|^{2}+\left(\mu-2 c_{6}\|\mathbf{w}\|\right)\|\nabla \mathbf{w}\|^{2} \\
& \quad \leq\left(\frac{1}{\mu}\left(\sup _{\Omega}\left|\mathbf{u}^{(2)}\right|\right)^{2}+2\left(\sup _{\Omega}\left|\nabla \mathbf{u}^{(2)}\right|\right)\right)\|\mathbf{w}\|^{2} .
\end{aligned}
$$

Therefore, on any interval during which $\|\mathbf{w}\| \leq \mu / 4 c_{6}$, making use of Poincaré's inequality (4) gives us

$$
\frac{d}{d t}\|\mathbf{w}\|^{2} \leq-c_{7}\|\mathbf{w}\|^{2}
$$

where

$$
c_{7}=\sup _{J}\left(\frac{\mu}{2 C_{\Omega}^{2}}-\frac{1}{\mu}\left(\sup _{\Omega}\left|\mathbf{u}^{(2)}\right|\right)^{2}-2\left(\sup _{\Omega}\left|\nabla \mathbf{u}^{(2)}\right|\right)\right),
$$

and $J$ is an interval of time during which $\|\mathbf{w}\| \leq \mu / 4 c_{6}$.

From Gronwall's inequality [6], we deduce that in the interval $J$

$$
\|\mathbf{w}\| \leq c \max _{J} \mathbf{w}_{0}, \quad \text { where } \mathbf{w}_{0}=\mathbf{u}_{0}^{(1)}-\mathbf{u}_{0}^{(2)} .
$$

Hence, a small change in the given data produces a correspondingly small change in the solution.

\section{References}

[1] L. Formaggia and C. Vergara, "Prescription of general defective boundary conditions in fluid-dynamics," Milan Journal of Mathematics, vol. 80, no. 2, pp. 333-350, 2012.

[2] J. G. Heywood, R. Rannacher, and S. Turek, "Artificial boundaries and flux and pressure conditions for the incompressible Navier-Stokes equations," International Journal for Numerical Methods in Fluids, vol. 22, no. 5, pp. 325-352, 1996.

[3] Y. A. Urzhumov and D. R. Smith, "Fluid flow control with transformation media," Physical Review Letters, vol. 107, no. 7, Article ID 074501, 2011.

[4] A. Quarteroni and A. Veneziani, "Analysis of a geometrical multiscale model based on the coupling of ODEs and PDEs for blood flow simulations," Multiscale Modeling \& Simulation. A SIAM Interdisciplinary Journal, vol. 1, no. 2, pp. 173-195, 2003.

[5] J. G. Heywood, "The Navier-Stokes equations: on the existence, regularity and decay of solutions," Indiana University Mathematics Journal, vol. 29, no. 5, pp. 639-681, 1980. 
[6] L. C. Evans, Partial Differential Equations, vol. 19 of Graduate Studies in Mathematics, American Mathematical Society, Providence, RI, USA, 2002.

[7] H. Fujita, "On the existence and regularity of the steady-state solutions of the Navier-Stokes equations," Journal of the Faculty of Science. University of Tokyo. Section IA. Mathematics, vol. 9, pp. 59-102, 1961.

[8] C. Guillopé and J.-C. Saut, "Existence results for the flow of viscoelastic fluids with a differential constitutive law," Nonlinear Analysis. Theory, Methods \& Applications A, vol. 15, no. 9, pp. 849-869, 1990. 


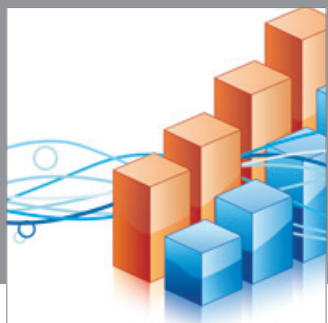

Advances in

Operations Research

mansans

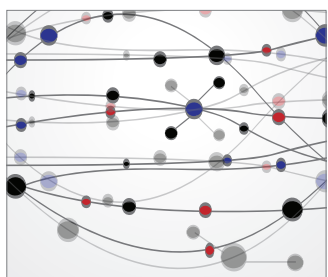

The Scientific World Journal
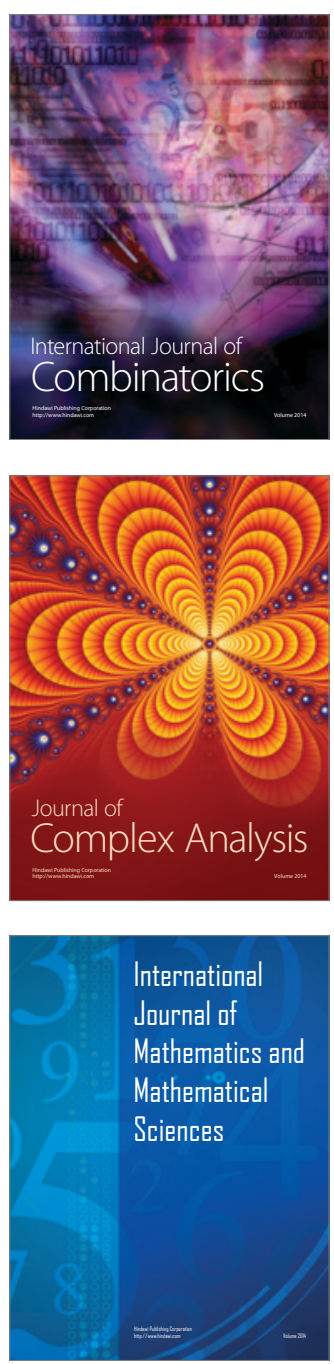
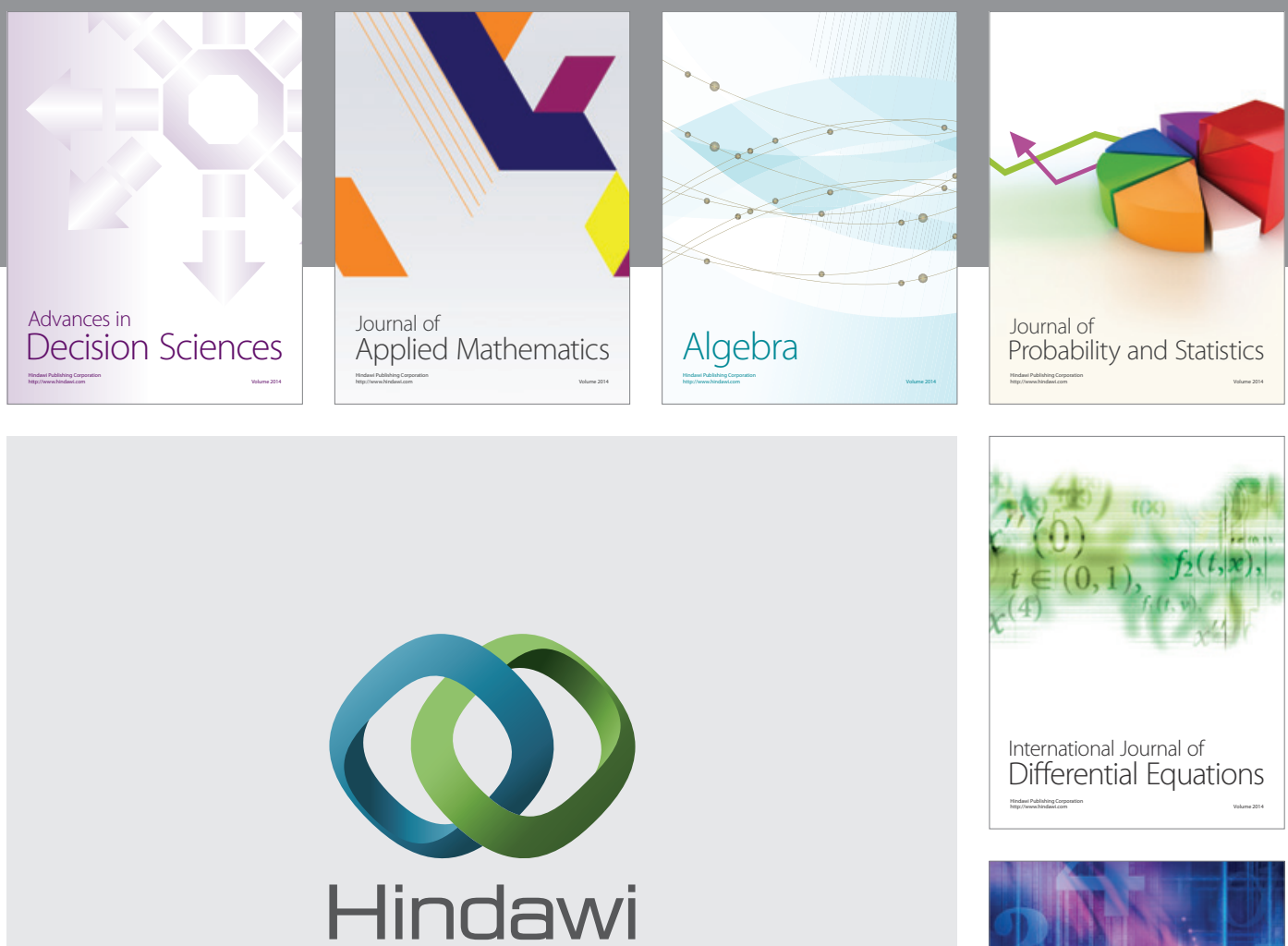

Submit your manuscripts at http://www.hindawi.com
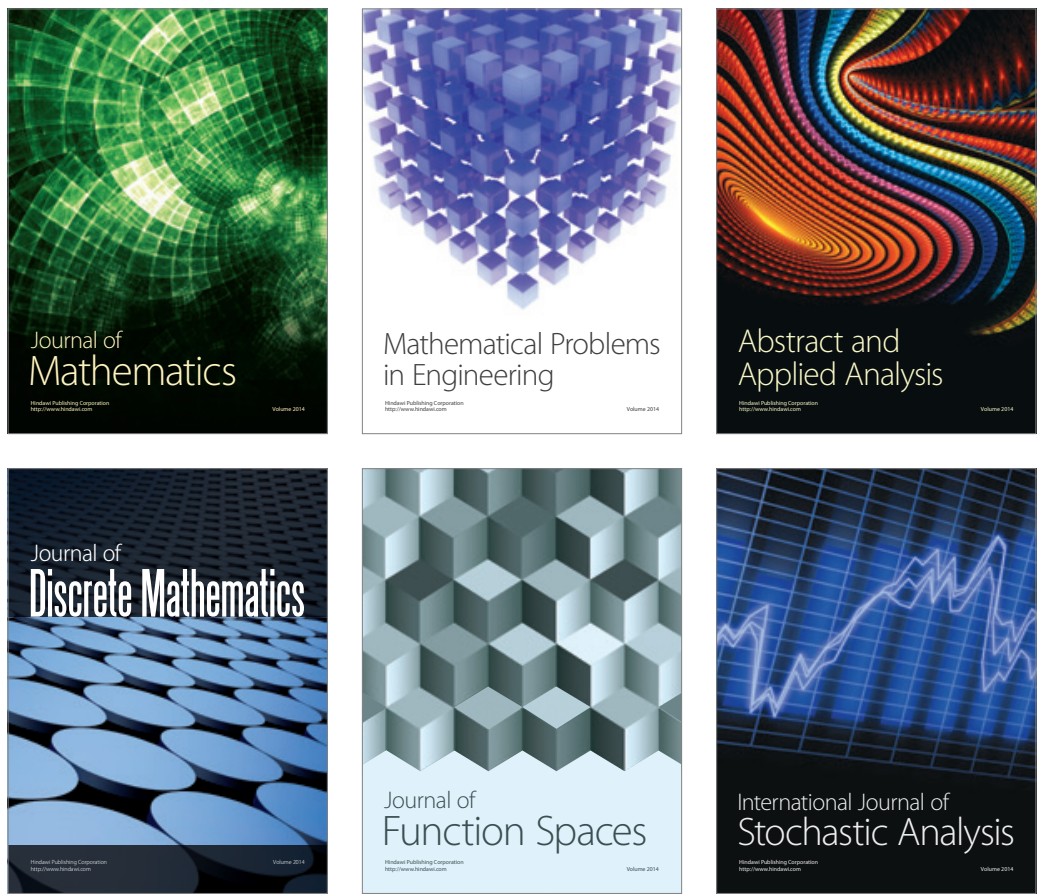

Journal of

Function Spaces

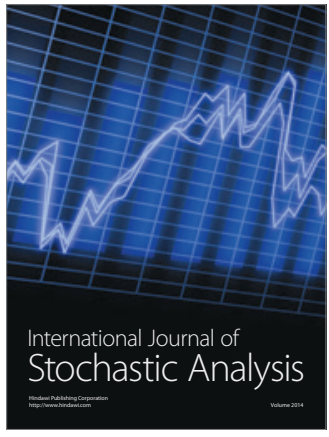

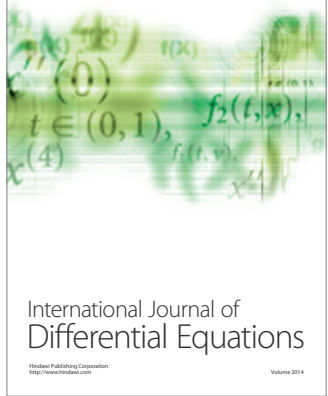
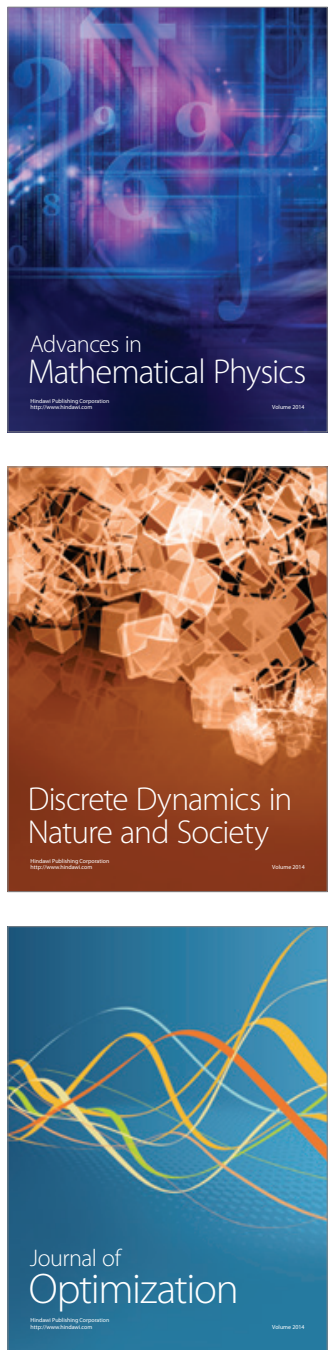